\title{
BDNF inhibits neurodegenerative disease- associated asparaginyl endopeptidase activity via phosphorylation by AKT
}

\author{
Zhi-Hao Wang, ${ }^{1,2}$ Wanqiang Wu, ${ }^{1,3}$ Seong Su Kang, ${ }^{1}$ Xia Liu, ${ }^{1}$ Zhiping Wu, ${ }^{4}$ Junmin Peng, ${ }^{4}$ Shan Ping Yu, ${ }^{5}$ \\ Fredric P. Manfredsson, ${ }^{6}$ Ivette M. Sandoval, ${ }^{6}$ Xuebo Liu, ${ }^{3}$ Jian-Zhi Wang, ${ }^{2}$ and Keqiang $\mathrm{Ye}^{1}$ \\ 'Department of Pathology and Laboratory Medicine, Emory University School of Medicine, Atlanta, Georgia, USA. \\ 2Department of Pathophysiology, Key Laboratory of Ministry of Education of Neurological Diseases, Tongji Medical College, \\ Huazhong University of Science and Technology, Wuhan, China. ${ }^{3}$ Laboratory of Functional Chemistry and Nutrition of \\ Food, College of Food Science and Engineering, Northwest A\&F University, Yangling, Shaanxi, China. ${ }^{4}$ Departments of \\ Structural Biology and Developmental Neurobiology, St. Jude Proteomics Facility, St. Jude Children's Research Hospital, \\ Memphis, Tennessee, USA. ${ }^{5}$ Department of Anesthesiology, Emory University School of Medicine, Atlanta, Georgia, USA. \\ ${ }^{6}$ Department of Translational Science \& Molecular Medicine, Michigan State University, Grand Rapids, Michigan, USA.
}

AEP is an age-dependent lysosomal asparaginyl endopeptidase that cleaves numerous substrates including tau and $\alpha$-synuclein and mediates their pathological roles in neurodegenerative diseases. However, the molecular mechanism regulating this critical protease remains incompletely understood. Here, we show that Akt phosphorylates AEP on residue T322 upon brain-derived neurotrophic factor (BDNF) treatment and triggers its lysosomal translocation and inactivation. When BDNF levels are reduced in neurodegenerative diseases, AEP T322 phosphorylation is attenuated. Consequently, AEP is activated and translocates into the cytoplasm, where it cleaves both tau and $\alpha$-synuclein. Remarkably, the unphosphorylated T322A mutant increases tau or $\alpha$-synuclein cleavage by AEP and augments cell death, whereas phosphorylation mimetic T322E mutant represses these effects. Interestingly, viral injection of T322E into Tau P301S mice antagonizes tau N368 cleavage and tau pathologies, rescuing synaptic dysfunction and cognitive deficits. By contrast, viral administration of T322A into young $\alpha$-SNCA mice elicits $\alpha$-synuclein N103 cleavage and promotes dopaminergic neuronal loss, facilitating motor defects. Therefore, our findings support the notion that BDNF contributes to the pathogenesis of neurodegenerative diseases by suppressing AEP via Akt phosphorylation.

Conflict of interest: The authors have declared that no conflict of interest exists.

Submitted: December 4, 2017

Accepted: July 3, 2018

Published: August 23, 2018

\section{Reference information:}

JCI Insight. 2018;3(16):e99007.

https://doi.org/10.1172/jci.

insight.99007.

\section{Introduction}

Neurodegenerative disease is a heterogeneous group of disorders characterized by progressive and selective loss of anatomically or physiologically related neuronal systems, with aging as the major risk factor. Classical examples include Alzheimer's disease (AD), Parkinson's disease (PD), amyotrophic lateral sclerosis (ALS), etc. (1). The pathological hallmark of filamentous tau inclusions, known as tauopathy (e.g., AD), is neurofibrillary tangles (NFTs) that consist of truncated and hyperphosphorylated tau. The pathological feature of synucleinopathies (e.g., PD) is $\alpha$-synuclein ( $\alpha$-syn) fibril aggregates that can be observed within neurons in the form of Lewy bodies (LBs), which primarily consist of truncated and phosphorylated $\alpha$-syn Most recently, we reported that mammalian asparaginyl endopeptidase (AEP; also known as legumain, $L G M N$ ) cleaves both APP and tau and mediates amyloid plaque formation and NFT pathologies in AD. AEP is upregulated and activated during aging, and it is highly activated in AD, cutting amyloid precursor protein (APP) at N373 and N585, yielding an APP (amino acids 586-695) fragment that is a better substrate for BACE1 to generate $A \beta$ peptide more efficiently. Hence, it acts as a $\delta$-secretase that binds and cleaves transmembrane APP in the endolysosomes (2). On the other hand, AEP also cuts tau at N255 and N368 and inhibits its microtubule-binding activity, promoting tau aggregation and neurotoxicity (3). Knockout of AEP or inhibition of AEP cleavage of APP or tau ameliorates the senile plaques and NFT pathologies in 5XFAD and Tau P301S mice, restoring cognitive functions $(2,3)$. Moreover, we found that AEP shreds human $\alpha$-syn at N103 and facilitates its aggregation, elevating neurotoxicity and triggering 
dopaminergic neuronal loss. AEP is also highly activated in PD brains versus healthy controls. Blockade of $\alpha$-syn cleavage by AEP or knockout of AEP attenuates the $\alpha$-syn pathologies in PD mouse models (4). Oral administration of small-molecule AEP inhibitor blunts APP or tau cleavage by AEP and prevents AD pathologies, rescuing the cognitive functions in various AD mouse models (5). Further, we have shown that AEP cleaves TDP-43, which is implicated in frontotemporal lobar degeneration (6). Previously, we have reported that AEP is involved in neuronal cell death by degrading the DNase inhibitor SET during excitoneurotoxicity (7). Notably, Iqbal et al. reported that AEP is highly upregulated and active in human $\mathrm{AD}$ brains and cleaves SET, a PP2A inhibitor, leading to tau hyperphosphorylation (8). They specifically proposed that AEP might serve as a therapeutic target for AD.

Mammalian AEP is an endolysosomal cysteine protease that cleaves after asparagine residues. AEP is distributed in all tissues but its abundance in the brain is low, where it is progressively elevated in an age-dependent manner (2-4). AEP activation is autocatalytic and requires sequential removal of C- and $\mathrm{N}$-terminal propeptides at different $\mathrm{pH}$ thresholds (9). Physiologically, AEP plays a pivotal role in the endolysosomal degradation system and is implicated in cathepsin cleavage and activation (10). The processing of the lysosomal proteases cathepsins in kidney is completely defective in AEP-deficient mice, with accumulation of macromolecules in the lysosomes (11). Moreover, we have previously reported that mice lacking AEP develop disorders resembling hemophagocytic syndrome (12). Interestingly, it also cleaves matrix metalloproteinase (MMP) proteins and mediates cancer metastasis (13). Most recently, we reported that the cell cycle-regulated kinase SRPK2 phosphorylates $\delta$-secretase (AEP) and enhances its enzymatic activity. SRPK2 phosphorylates serine 226 on AEP and accelerates its autocatalytic cleavage, leading to its cytoplasmic translocation and augmented enzymatic activities. Accordingly, AEP is highly phosphorylated on S226 in human AD brains, which is tightly correlated with SRPK2 activity (14).

Mounting evidence supports the notion that alterations in brain neurotrophic support and in particular in brain-derived neurotrophic factor (BDNF) expression and signaling might contribute to neurodegeneration (15). Neurotrophins are growth factors that regulate neuronal development, differentiation, and survival in both peripheral and central nervous systems. Neurotrophins regulate neurotrophic functions through binding with their specific cell surface receptors. For instance, BDNF and NT-4/5 bind TrkB. Neurotrophins bind Trk receptors and trigger the receptor dimerization and activation of numerous downstream signaling cascades, including Ras/Raf/MAPK, PI3K/Akt, and PLC- $\gamma 1$, which play critical roles in neuronal plasticity, survival, neurogenesis, etc. (16). BDNF is essential for long-term potentiation (LTP) induction and maintenance and long-term memory (17-19). Altered functionality of BDNF has been observed in different neurodegenerative diseases $(20,21)$. A reduction of BDNF mRNA and protein expression has been consistently reported in multiple brain areas of $\mathrm{AD}$ postmortem and in the substantia nigra (SN) of $\mathrm{PD}$ patients $(22,23)$. Further, serum BDNF levels are also reduced in some of the neurodegenerative diseases (24). Interestingly, neurons containing NFTs do not contain BDNF immunoreactivity, whereas intense BDNF-positive neurons are devoid of tangles (25). These studies suggest that BDNF may have a protective role against $\mathrm{AD}$ pathogenesis. The beneficial effect of BDNF administration has been shown to improve learning and memory of demented animals (26). Studies in AD models show that BDNF has a neuroprotective effect against A-amyloid toxicity (27). Accordingly, BDNF gene delivery has been shown as a novel potential therapeutic approach in diverse models related to $\mathrm{AD}(28)$. On the other hand, loss of BDNF production has been associated with mutations of $\alpha$-syn in early-onset familial PD (29). Expressing BDNF in either the striatum or the midbrain attenuates 6-hydroxydopamine -induced (6-OHDA-induced) loss of nigrostriatal neurons, promoting behavioral improvements (30-32). Moreover, BDNF prevents MPTP (1-methyl-4-phenyl-1,2,3,6-tetrahydropyridine) or 6-OHDA toxicity to dopaminergic neurons in rats (31, 33). Together, these reports strongly support the idea that BDNF reduction might contribute to the pathogenesis of neurodegenerative diseases. In the current study, we show that BDNF-mediated Akt directly phosphorylates AEP on T322 and blocks AEP activation, sequestering it in lysosomes. BDNF reduction in neurodegenerative diseases elicits reduction of phosphor-AEP (p-AEP) T322, AEP cytoplasmic translocation, and subsequent activation, escalating its autocleavage and pathological substrate truncation. Viral delivery of phosphorylation mimetic T322E mutant into the hippocampus prevents tau N368 cleavage in Tau P301S-Tg mice, ameliorating the NFT pathologies and cognitive deficits. Viral administration of unphosphorylated T322A mutant into the SN in young $\alpha$-SNCA mice accelerates $\alpha$-syn N103 cleavage and LB pathology in $\alpha$-SNCA-Tg mice, promoting early dopaminergic loss and motor dysfunctions. 


\section{Results}

Akt phosphorylates AEP on residue T322. Apoptotic caspases cleave AEP at D25 and upregulate its maturation and activation (9). Since Akt is a major prosurvival downstream effector of BDNF/TrkB neurotrophic signaling, we hypothesized that Akt might directly phosphorylate AEP and mitigate its deleterious enzymatic activity. To test this hypothesis, we conducted an in vitro Akt phosphorylation assay with purified recombinant AEP proteins. Clearly, Akt robustly phosphorylated AEP and the positive control tau (Figure $1 \mathrm{~A}$ ). In HEK293 cells cotransfected with various HA-tagged Akt constructs and glutathione S-transferase-tagged AEP (GST-AEP), the transfected AEP protein was pulled down with glutathione beads and analyzed with an anti-p-Akt-substrate antibody, which nonselectively recognizes Akt-phosphorylated substrates. Noticeably, AEP was robustly phosphorylated by wild-type (WT) or constitutively active (CA) Akt, with CA signal stronger than WT; by contrast, AEP was not phosphorylated in the presence of kinase-dead (KD) Akt (Figure 1B). Akt autophosphorylation also correlated with this pattern (Figure 1B). To map the potential phosphorylation site, we cotransfected different AEP fragments with WT Akt. The truncate aa 301-433 was strongly phosphorylated in addition to full-length (FL) AEP (Figure 1C). Thus, Akt might phosphorylate AEP on the C-terminal 301-433 tail. Proteomic analysis with phosphorylated recombinant AEP proteins demonstrated that T322 was selectively phosphorylated by Akt (Figure 1D). Mutation of T322 to A322 abolished Akt in vitro phosphorylation of AEP (Figure 1E). To confirm that T322 is the genuine site on AEP phosphorylated by Akt, we developed a p-T322-specific polyclonal antibody. The affinity columnpurified antibody specifically recognized WT but not T322A AEP, which was abolished in the presence of peptide antigen (Supplemental Figure 1A; supplemental material available online with this article; https:// doi.org/10.1172/jci.insight.99007DS1), indicating that this antibody selectively recognized p-AEP T322 pulled down by glutathione beads. Moreover, this antibody also specifically recognized WT AEP but not T322A in the transfected cell lysates, although both were equally overexpressed (Supplemental Figure 1B). Since AEP is highly expressed in the kidney cell line HEK293, we extended our characterization to endogenous AEP. In HEK293 cells transfected with various HA-tagged Akt constructs, endogenous AEP protein was pulled down and analyzed with anti-p-Akt-substrate antibody. AEP was robustly phosphorylated by CA Akt and not in KD Akt (Supplemental Figure 1C). EGF-provoked AEP phosphorylation on T322 was selectively blocked by PI3K or Akt inhibitor (wortmannin or Akt1/2i) (Supplemental Figure 1D), supporting the idea that anti-p-T322 antibody explicitly identified the Akt-phosphorylated AEP. We made similar observations in primary neurons. BDNF strongly provoked Akt activation, which phosphorylated AEP on T322 that could be diagnosed by both p-Akt-substrate and p-AEP-T322 antibodies. This phosphorylation was abrogated by Trk inhibitor K252a, PI3K inhibitor wortmannin, and LY294002, or Akt inhibitor but not MEK inhibitor PD98059 (Supplemental Figure 1E), suggesting that BDNF triggers AEP T322 phosphorylation via activating Akt. To validate that residue T322 is indeed the authentic Akt phosphorylation site on AEP, we generated several $\mathrm{S} / \mathrm{T} \rightarrow \mathrm{A}$ mutants within the pseudo-Akt-phosphorylation motif. As expected, anti-p-T322 antibody failed to distinguish the T322A mutant, whereas it potently recognized all the other mutants (Figure 1F). Remarkably, this antibody unambiguously identified p-AEP T322 in WT but not $L G M N$-deficient mouse brains (Figure $1 \mathrm{G}$ ), underscoring that endogenous AEP is phosphorylated by Akt in the brain.

AEP is glycosylated and this modification mediates its sorting from the ER into lysosomes $(34,35)$. To examine whether transfected GST-AEP is indeed glycosylated, we transfected GST-AEP into HEK293 cells, followed by treatment with tunicamycin, an inhibitor of N-linked glycosylation, and PNGase F and Endo $\mathrm{H}$ were used to characterize the glycosylation types. Immunoblotting showed that glycosylated 75-kDa GST-AEP was reduced after tunicamycin treatment, suggestive of deglycosylation of GST-AEP. Endo H, but not PNGase F, evidently deglycosylated GST-AEP (Supplemental Figure 2).

$B D N F$ triggers Akt-phosphorylated AEP lysosomal translocation. AEP is a lysosomal cysteine protease (36). To assess the effect of Akt phosphorylation of AEP T322 on its subcellular residency, we conducted immunofluorescent staining on HEK293 cells transfected with GFP fused with AEP WT, T322A, or T322E, in which the signal peptide of AEP was upstream of the GFP tag (C-terminal GFP tag). LAMP1 and TOMM20 were employed as markers for the lysosomes and mitochondria, respectively. Phosphorylation mimetic T322E predominantly colocalized with LAMP1; in contrast, WT or T322A exhibited less colocalization with LAMP1, indicating that Akt phosphorylation of AEP induces its lysosomal translocation. AEP barely colocalized in TOMM20-positive mitochondria (Supplemental Figure 3A). We made a similar observation with endogenous AEP using anti-p-T322 antibody in primary neurons (Supplemental 


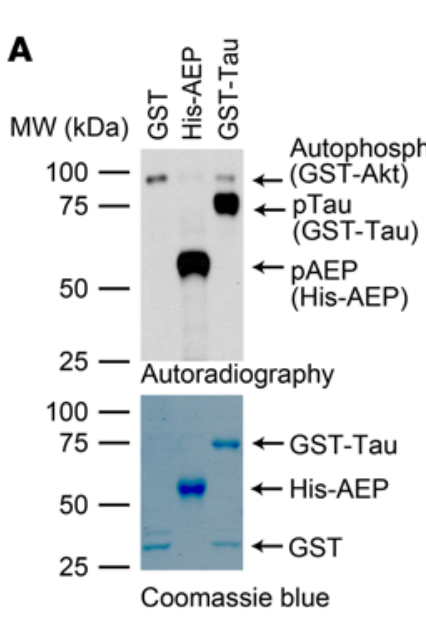

D

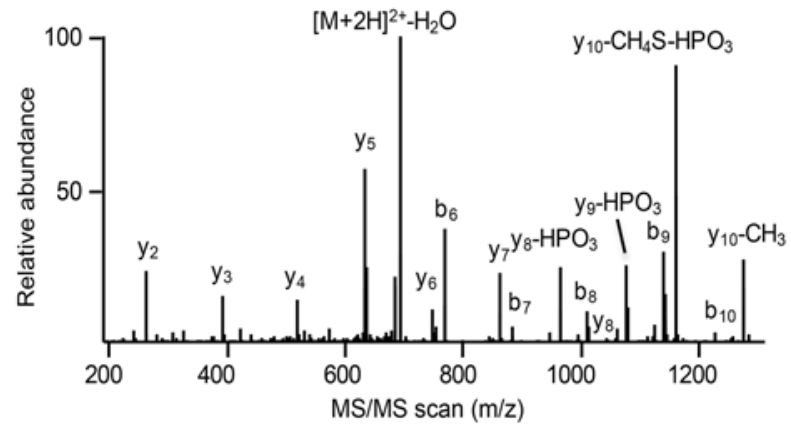

E

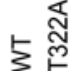

s.

岸岸

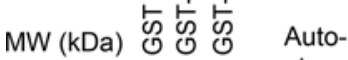

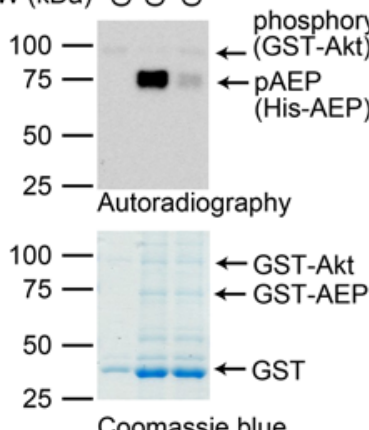

Coomassie blue

75 -

$75-$ Cell lysate

$b_{6} b_{7} b_{8} b_{9} b_{10}$

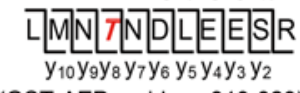

(GST-AEP residues 319-329)

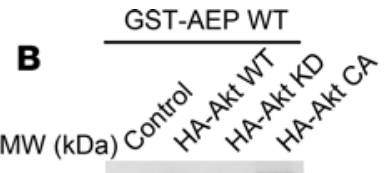

GST pull-down

WB: anti-phospho-

Akt-substrate

$75-\infty \omega \infty$

GST pull-down

WB: anti-GST

WB: anti-GST

$75-\underset{\text { Cell lysate }}{-}$

WB: anti-Akt pS473

$75-1$ - Cell lysate
WB: anti-HA

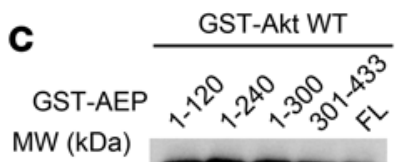

MW (kDa)
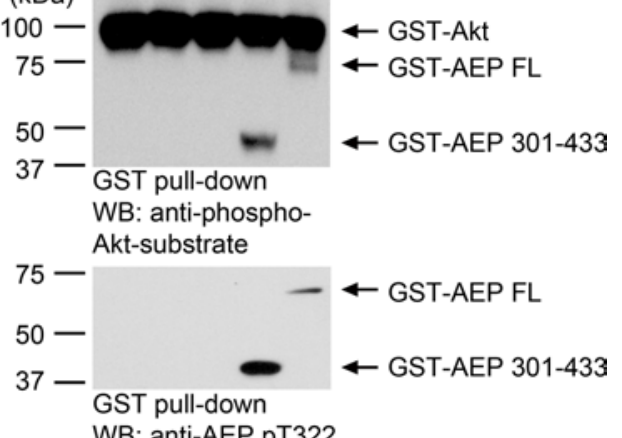

100 —

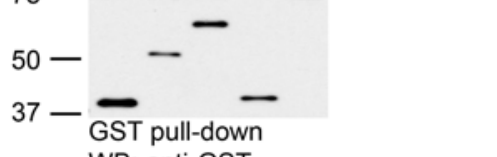

WB: anti-GST

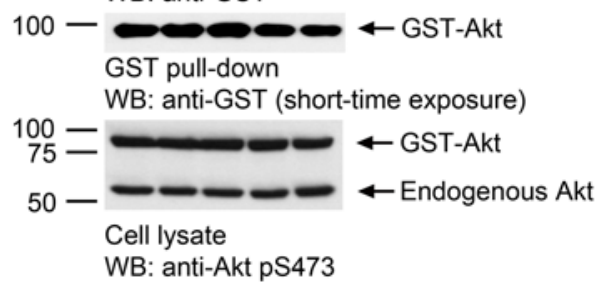

10-month-old mouse

brain lysate

G
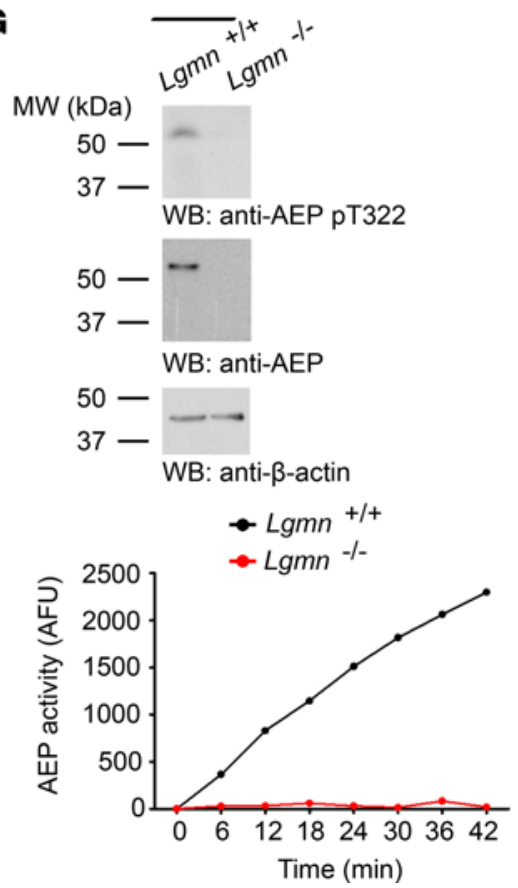


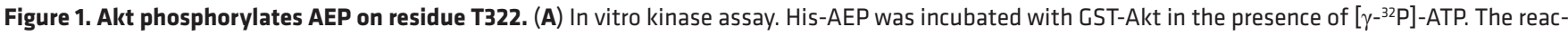
tion products were separated in SDS-PAGE and analyzed by autoradiography. GST and GST-tau were used as negative and positive control, respectively. The protein inputs are shown in the lower panel. (B) Akt phosphorylates AEP in intact cells. Mammalian GST-AEP was cotransfected with HA-Akt WT, KD, or CA into HEK293 cells. At 48 hours, GST-AEP was pulled down and analyzed with anti-p-Akt substrate antibody (first panel) and anti-CST antibody (second panel). Expression of GST-AEP (third panel) and HA-tagged p-/total Akt (fourth and fifth panel) in cell lysates are also shown. (C) Western blot showing phosphorylation of full-length and fragmented AEP by Akt in HEK293 cells. (D) MS/MS spectrum showing the phosphorylation of AEP on residue T322 in GST-AEP purified from HEK293 cells that were cotransfected with GST-AEP and HA-Akt. (E) T322 in AEP is the major phosphorylation residue. In vitro kinase assay showing phosphorylation of WT and mutated AEP ( $T$ to $A$, unphosphorylated mutant) by Akt (upper panel). The inputs are shown in the lower panel. (F) Western blot analysis of phosphorylation level of WT and various mutated AEP constructs after cotransfection with Akt. (C) Knockout of AEP abrogated p-T322 antibody immunoblotting signals on AEP. AFU, arbitrary fluorescence units.

Figure 3B). Moreover, BDNF elicited pronounced AEP T322 phosphorylation, colocalizing with AEP (Supplemental Figure 3C). On the other hand, A $\beta$ decreased p-AEP T322, reducing its colocalization with LAMP1 (Supplemental Figure 3D). Immunofluorescent costaining assays in neurons showed that BDNF treatment evidently augmented p-AEP T322 and p-Akt S473 signals, and p-T322 tightly colocalized with Akt in the cytoplasm, as did p-Akt S473 with AEP (Figure 2, A and B). We made similar observations in BR6 cells stably transfected with TrkB, which were abolished by Akt inhibitor, evidence that BDNFpromoted phosphorylation of AEP T322 is mediated by Akt. However, in TrkB-deficient SH-SY5Y cells, neither AEP nor Akt phosphorylation was responsive to BDNF stimulation (Supplemental Figure 3E). By contrast, A $\beta 42$ peptide dose-dependently decreased both p-T322 and p-Akt S473 intensities, diminishing their colocalization in the neuronal cytoplasm (Figure 2, C and D). Quantification analysis supported these observations (Figure 2, E-H). To further dissect the role of BDNF in AEP subcellular residency, we conducted subcellular fractionation using SH-SY5Y and BR6 cells. BDNF induced demonstrable Akt activation in BR6 but not SY5Y cells. Remarkably, BDNF-activated p-Akt S473 localized in both the lysosomal and cytosolic fractions in BR6 cells, whereas the basal p-Akt S473 only distributed in the cytosolic fraction. Consequently, AEP was robustly phosphorylated on T322 by Akt in BR6 cells. p-AEP was mostly distributed in the lysosomes. The truncated and mature $37-\mathrm{kDa}$ form of AEP specifically resided in the lysosomes and BDNF stimulation reduced its cytosolic distribution, with repression of its fragmentation in the lysosomes of BR6 but not SY5Y cells. By contrast, Akt was more abundant in the cytosolic fraction than the lysosomal fraction (Figure 2I). These findings indicate that BDNF via TrkB receptors triggers Akt activation, which subsequently phosphorylates AEP and induces its lysosomal translocation. Time course assay with BR6 cells revealed that BDNF temporally induced p-AEP T322 increases in both the cytosolic and lysosomal fractions, which were completely blunted by Akt inhibitor or Akt knockdown. Accordingly, AEP cleavage in both fractions was time-dependently inhibited upon BDNF treatment. However, in the presence of Akt inhibitor or Akt siRNA, the lysosomal AEP cleavage was demonstrable and its cytosolic fragmentation was modest (Figure 2J and Supplemental Figure 3F). Notably, p-Akt S473 echoed the p-AEP T322 activation patterns, both of which were robustly antagonized by Akt1/2i (Figure 2J). Thus, these data support the notion that BDNF-activated Akt phosphorylates AEP and triggers its lysosomal translocation, suppressing its activation.

$B D N F /$ TrkB reduction in neurodegenerative diseases elicits AEP cytosolic residency and activation. To assess whether $\mathrm{BDNF} / \mathrm{TrkB}$ signaling is required for mediating AEP phosphorylation, we prepared primary mouse neurons from $\operatorname{Trk}^{f / f l}$ mice, and infected the cultures with GFP or Cre virus. In TrkB $B^{f / f l}$ neurons, endogenous TrkB was substantially depleted by Cre recombinase compared with GFP control, and p-TrkB and p-Akt were consequently decreased, as was p-AEP T322 (Supplemental Figure 4A). AEP enzymatic activities in TrkB-knockout neurons tightly coupled with its cleavage activities (Supplemental Figure 4B). Next, we monitored both p-AEP T322 and p-Akt S473 in the brain sections of $\mathrm{AD}$ and $\mathrm{PD}$, as BDNF and its TrkB receptors are reduced in these neurodegenerative diseases. Both phosphorylation activities were reduced in these brains. Inversely, the total AEP expression levels were augmented (Figure 3, A-H), suggesting that BDNF/TrkB reduction in neurodegenerative diseases elicits AEP upregulation and its p-T322 attenuation in the brain. Immunoblotting analysis validated that $\mathrm{p}$-TrkB and p-Akt signaling was substantially reduced in the hippocampus of $\mathrm{AD}$ brains as compared with healthy controls, as was p-AEP T322. In contrast, AEP was highly increased in AD brains, which was associated with active fragmentation (Figure 4, A and C). AEP was inversely activated in AD brains, with the cytosolic fraction more robust than the lysosomal fraction (Figure 4B). In synucleinopathies like DLB (dementia with Lewy body), we also found that reduced BDNF/p-TrkB/p-Akt signaling correlated with diminished p-AEP T322, and inversely coupled with AEP fragmentation and activation (Figure 4, D and E). Together, these data strongly 
A

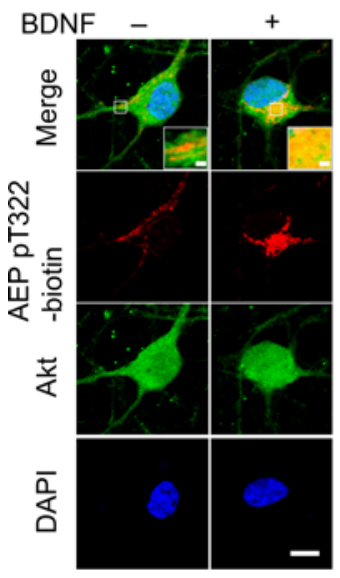

E

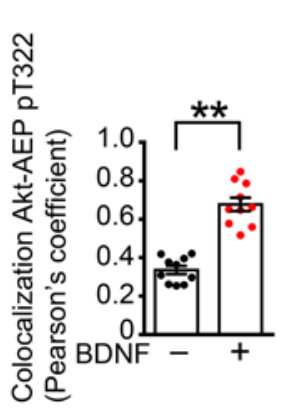

B

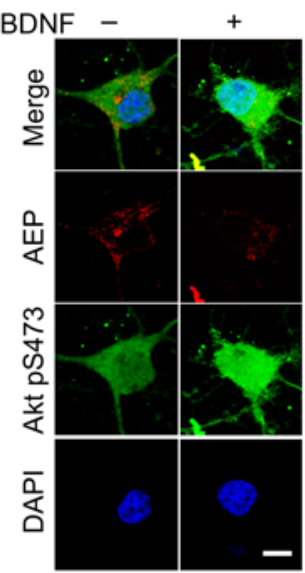

F

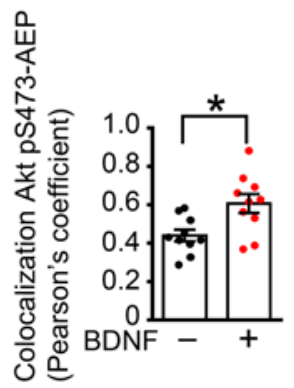

C

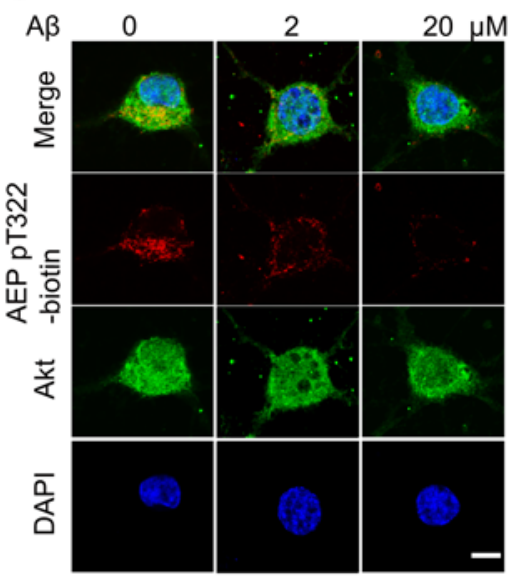

G

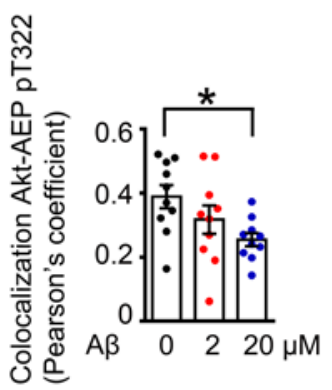

D

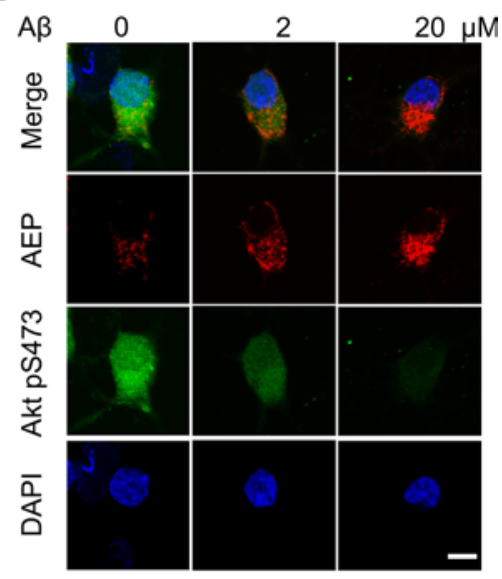

H

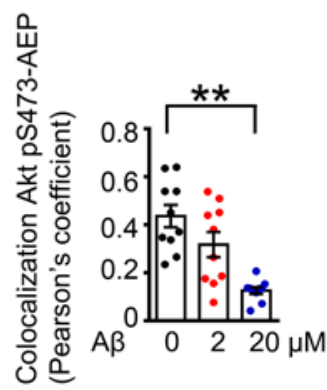

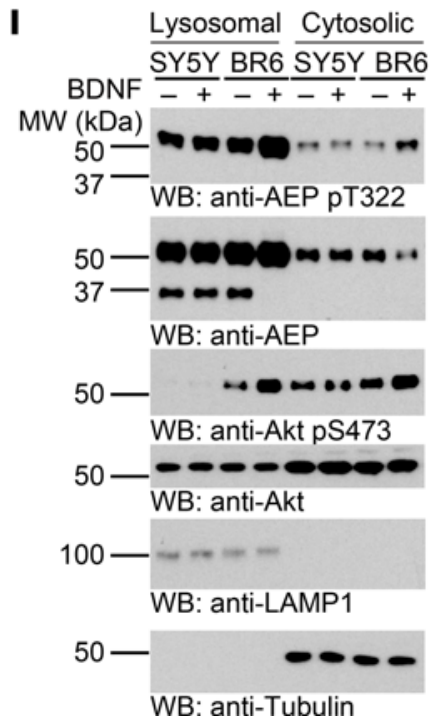

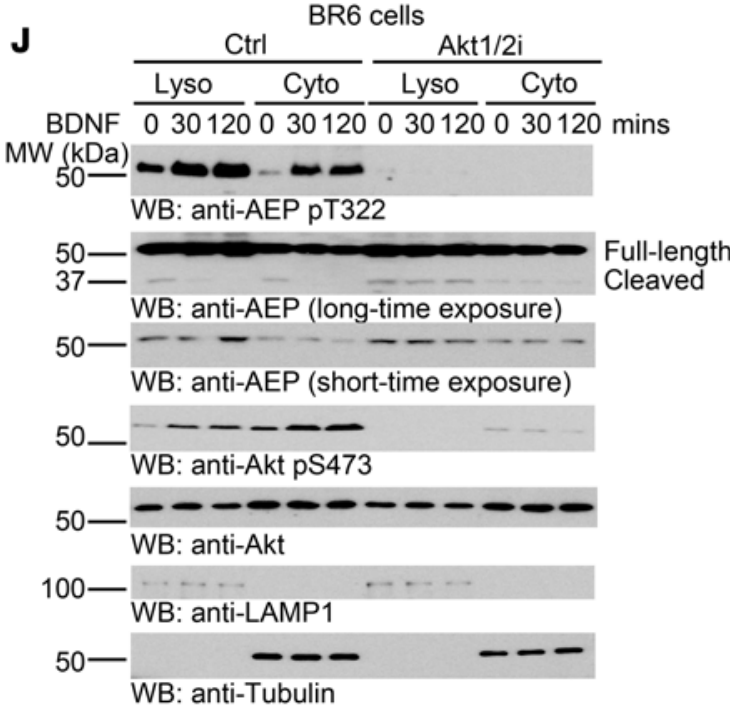

Figure 2. BDNF triggers Akt-phosphorylated AEP lysosomal translocation. (A and B) Colocalization between AEP pT322/Akt (A) and Akt pS473/ AEP (B) in rat primary cortical neurons (DIV 13) treated with or without $100 \mathrm{ng} / \mathrm{ml}$ BDNF for 30 minutes. (C and D) Colocalization between AEP pT322/Akt (C) and Akt pS473/AEP (D) in rat primary cortical neurons (DIV 13) treated with 2 or $20 \mu$ M preaggregated A $\beta$ for 16 hours. p-AEP T322 was biotinylated. Scale bars: $5 \mu \mathrm{m}$. (E-H) Quantification of colocalization in A-D. Data shown as the mean \pm SEM ( $n=10$ cells per group). ${ }^{*} P<0.05$; ${ }^{*} P<0.01$ by 2-tailed $t$ test for $\mathbf{E}$ and $\mathbf{F}, 1$-way ANOVA with Tukey's multiple-comparisons test for $\mathbf{G}$ and $\mathbf{H}$. (I) $p$-T322 mediates AEP lysosomal translocation. Cells were treated with $100 \mathrm{ng} / \mathrm{ml} \mathrm{BDNF}$ for 30 minutes, and then were subjected to subcellular fractionation. LAMP1 was used as specific marker for the lysosomal fraction. Tubulin was used as a loading control for the cytoplasmic fraction. (J) Akt phosphorylation translocates cytoplasmic AEP into the lysosomes. BR6 cells were treated with control or Akt inhibitor $(10 \mu \mathrm{M})$, followed by BDNF (100 ng/ml) treatment at different time points. Western blot data in I and $\mathbf{J}$ are representative of 3 independent experiments. 
A
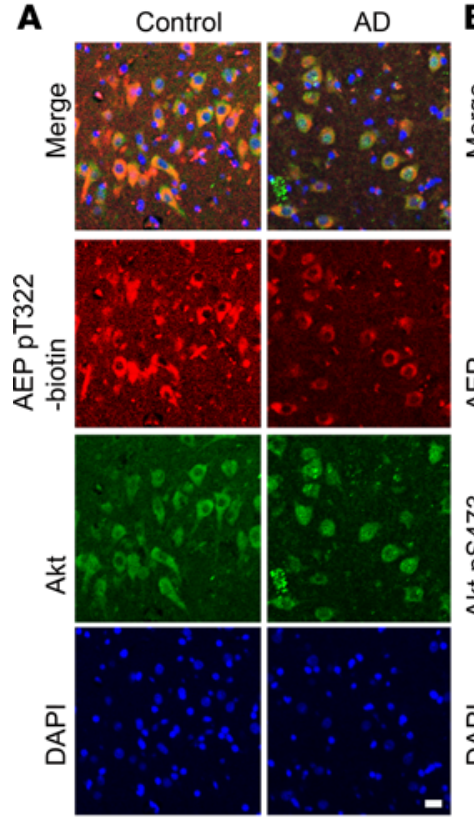

E

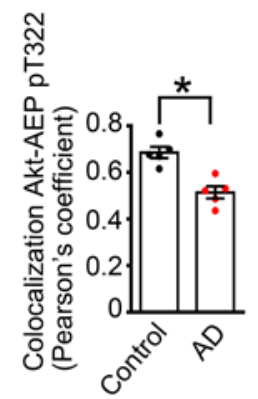

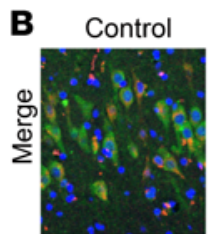
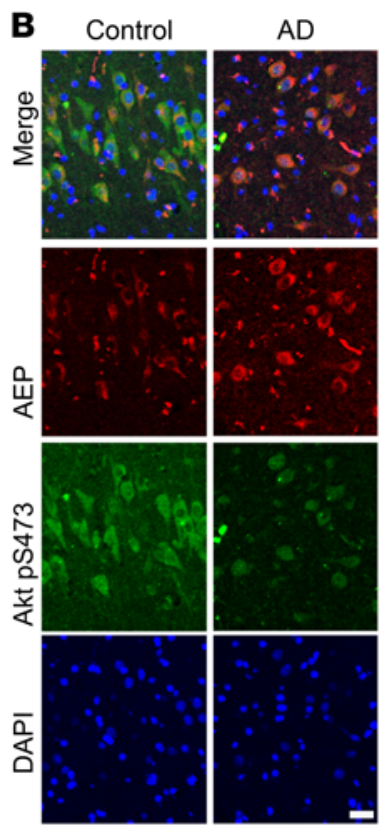

$\mathbf{F}$

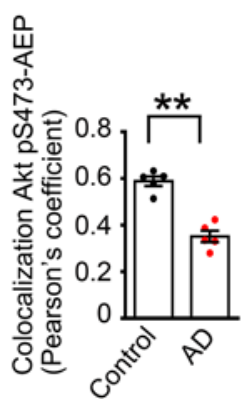

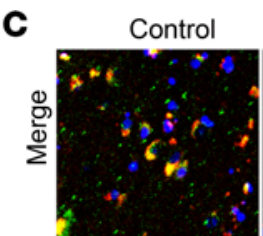
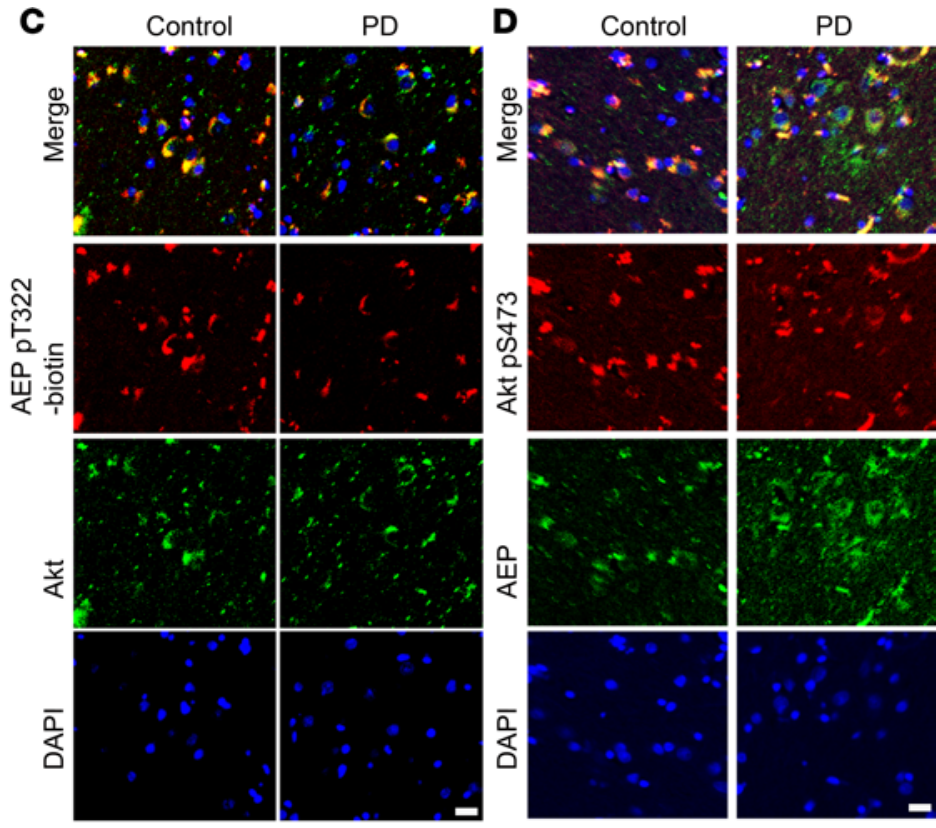

G

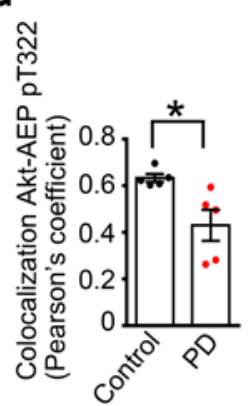

H

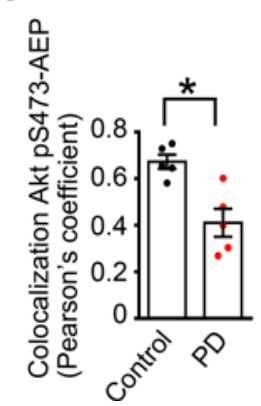

Figure 3. AEP phosphorylation by Akt is inhibited in neurodegenerative diseases. (A-D) Immunofluorescent staining of Akt/AEP pT322 and Akt pS473/AEP in human AD hippocampal CA1 (A and B) and PD substantia nigra (C and D) compared with age-matched healthy control. Confocal imaging shows colocalization. Scale bars: $10 \mu \mathrm{m}$. (E-H) Quantification of colocalization in A-D. Data shown as the mean \pm SEM ( $n=5$ cases per group). ${ }^{*} P<0.05,{ }^{*} P<0.01$ by 2 -tailed $t$ test.

support the hypothesis that a decrease in BDNF/TrkB signaling in neurodegenerative diseases abolishes p-AEP $\mathrm{T} 322$, resulting in AEP upregulation and activation.

Akt phosphorylation of AEP inhibits its autocleavage and enzymatic activation. To assess the biological consequence of Akt phosphorylation of AEP, we examined AEP enzymatic activity in the cells cotransfected with GST-AEP and HA-tagged WT, KD, or CA Akt. Interestingly, CA Akt displayed the strongest effect in repressing AEP enzymatic activity, followed by WT Akt. By contrast, AEP exhibited comparable activities in the presence of control and $\mathrm{KD}$ Akt, indicating that Akt phosphorylation of AEP may suppress its enzymatic activity (Figure 5A). This notion was confirmed in phosphorylation mimetic T322E and the unphosphorylated T322A mutant (Figure 5B). Quantitative AEP enzymatic assay demonstrated that only the T322A mutant exhibited the strongest enzymatic activity among WT AEP and all other mutants in the presence of Akt, indicating that the T322A mutation is resistant to Akt phosphorylation-induced enzymatic activity suppression (Supplemental Figure 5A). Immunoblotting analysis revealed the timedependent fragmentation of AEP, with T322A being the strongest, followed by WT, and T322E was barely cleaved (Figure 5C). Quantitative analysis of the relative ratios of the fragment versus FL AEP supported this finding (Figure 5C). In addition to AEP autocleavage, we also found similar enzymatic activities for 2 well-characterized substrates: tau and $\alpha$-syn (Figure 5, D and E). To further investigate the effect of Akt phosphorylation of AEP on its autocleavage and enzymatic cleavage of tau and $\alpha$-syn, we incubated GSTtagged AEP WT or T322A with HA-conjugated WT or KD Akt for 2 hours. We then initiated autocleavage 
A

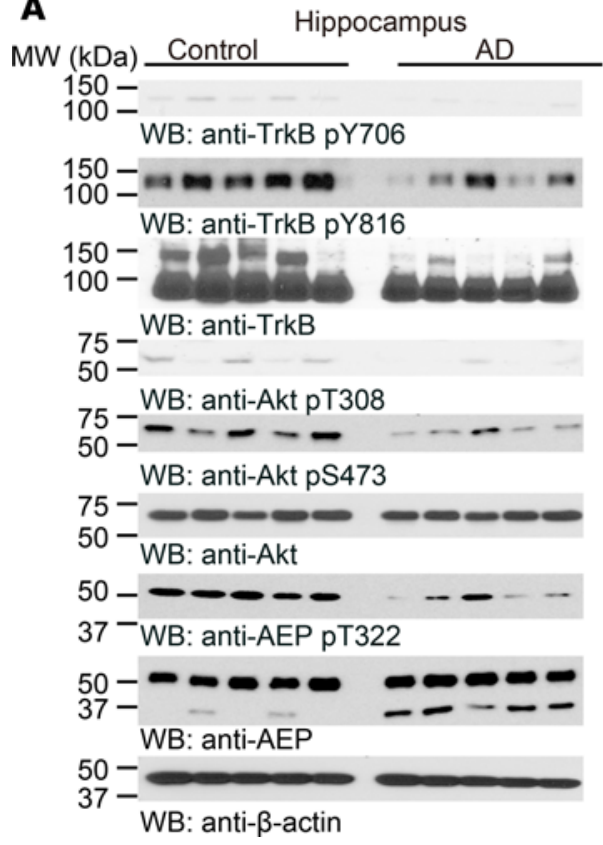

B Human brain

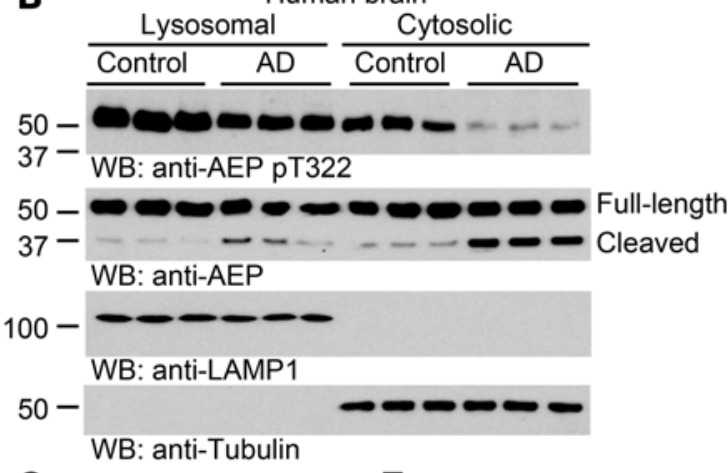

C

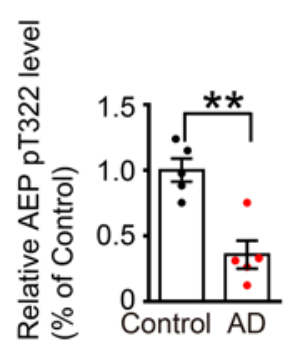

$\mathbf{E}$

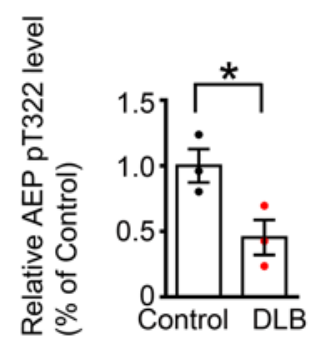

Figure 4. BDNF reduction in neurodegenerative diseases elicits AEP cytosolic residency and activation. (A-E) Western blot analysis of phosphorylated $A E P$ and $A k t$ in $A D(A-C)$ or DLB brains ( $\mathbf{D}$ and $\mathbf{E}$ ) compared with age-matched healthy controls. Western blot data are representative of 3 independent experiments. Data in $\mathbf{C}$ and $\mathbf{E}$ shown as the mean $\pm \operatorname{SEM}\left(n=3-5\right.$ per group). ${ }^{*} P<0.05,{ }^{* *} P<0.01$ by 2-tailed $t$ test.

and substrate fragmentation in $\mathrm{pH} 6.0$ buffer at different time points. Remarkably, WT AEP but not T322A displayed a time-dependent autocleavage regardless of WT or KD Akt (Supplemental Figure 5B). AEPtruncated tau N368 and $\alpha$-syn N103 abundance oscillated with AEP autocleavage activity (Supplemental Figure 5B). Hence, T322 phosphorylation by Akt inhibits AEP activation, preventing AEP from cleaving its pathological substrates.

Akt-phosphorylated AEP blocks $\alpha$-syn-or tau P301S-induced cell death. AEP mediates neuronal cell death under acidosis triggered by hypoxia or excitotoxicity (7). To test whether Akt phosphorylation of AEP affects its pro-cell death activity, we used BR6 dopaminergic cells stably transfected with TrkB and cotransfected them with GST-tagged AEP WT, T322A, or T322E and tau P301S or AEP-uncleavable tau P301S/ N255A/N368A, followed by BDNF treatment. Immunoblotting revealed that endogenous tau in BR6 was cleaved by WT AEP, which was repressed by BDNF. By contrast, T322A robustly cleaved tau N368, which was resistant to BDNF. Nonetheless, T322E failed to cut tau at N368. Tau P301S (untagged) was evidently truncated as validated by Tau- 5 antibody and it displayed a response to BDNF similar to that of tau N368. It is worth noting that the truncated tau fragments in the presence of tau P301S were much more pronounced than with the control. By contrast, with the AEP-resistant tau mutant, neither Tau-5 nor tau N368 antibody detected any demonstrable fragment from overexpressed tau (Figure 6A). Lactate dehydrogenase (LDH) assay showed that WT AEP-induced cell death was significantly inhibited by BDNF in the control cells. Overexpression of tau P301S induced more robust cell death, which was enhanced by WT AEP, and the peak activity occurred on T322A. The cell death with T322E was significantly blunted as compared with WT AEP. However, the AEP-uncleavable tau mutant increased the cell death baseline and the cell death was not affected by BDNF regardless of whether WT or phosphorylation-mutant AEP was transfected (Figure 6B). Presumably, the AEP-uncleavable tau mutant triggered cell death via a mechanism that was independent of BDNF/Akt signaling. We made a similar observation with $\alpha$-syn N103 cleavage by WT AEP, which was blocked by BDNF. Notably, $\alpha$-syn N103 truncation patterns tightly coupled with tau N368 cleavage, consistent with our previous reports that AEP simultaneously mediates both substrates' fragmentation $(3,4)$. Again, T322A exhibited the strongest protease activity, whereas T322E possessed no endopeptidase activity, and was not responsive to BDNF treatment (Figure 6C). Cell death assay results were consistent with $\alpha$-syn N103 fragmentation intensities. GFP- $\alpha$-syn N103A triggered cell death with a higher baseline than that of control (Figure 6D), consistent with $\alpha$-syn neurotoxicity in dopaminergic cells. 


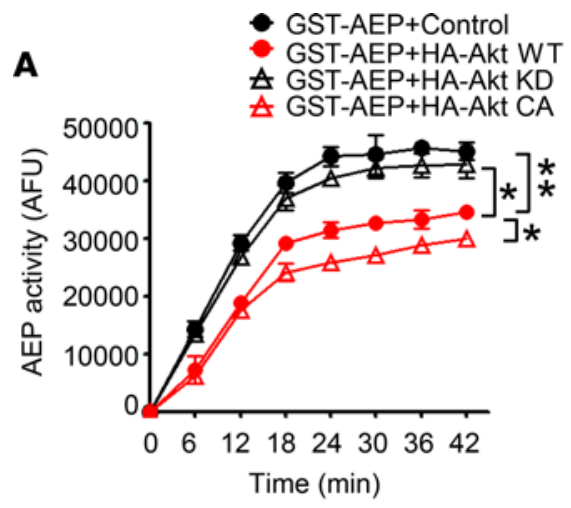

C

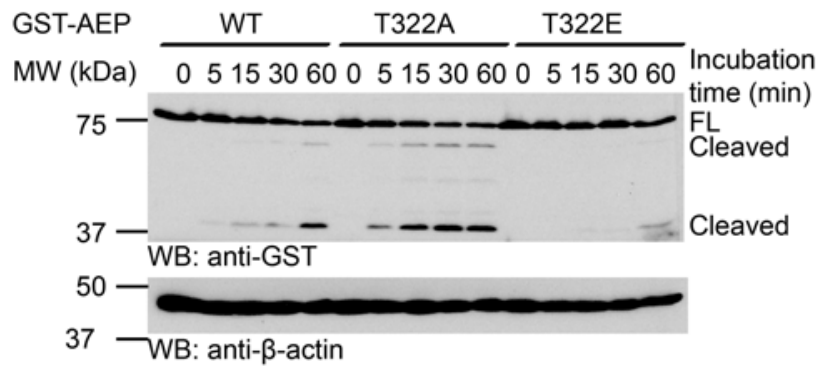

D

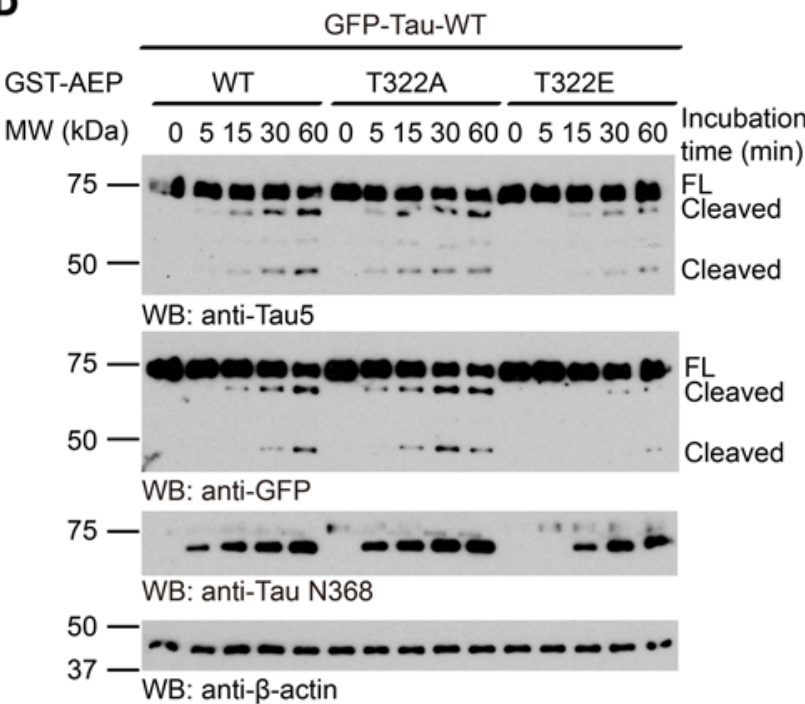

E

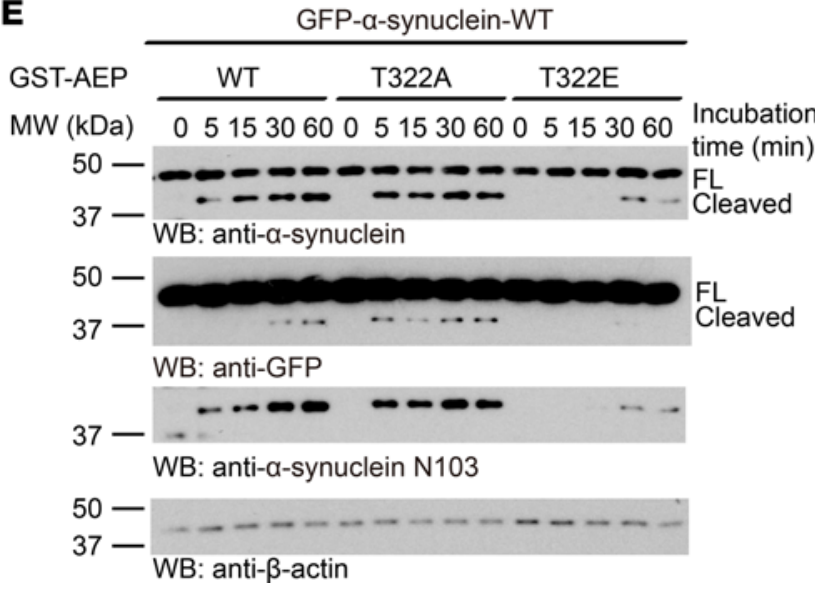

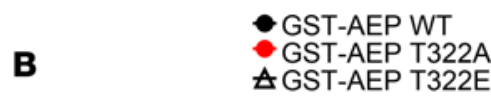
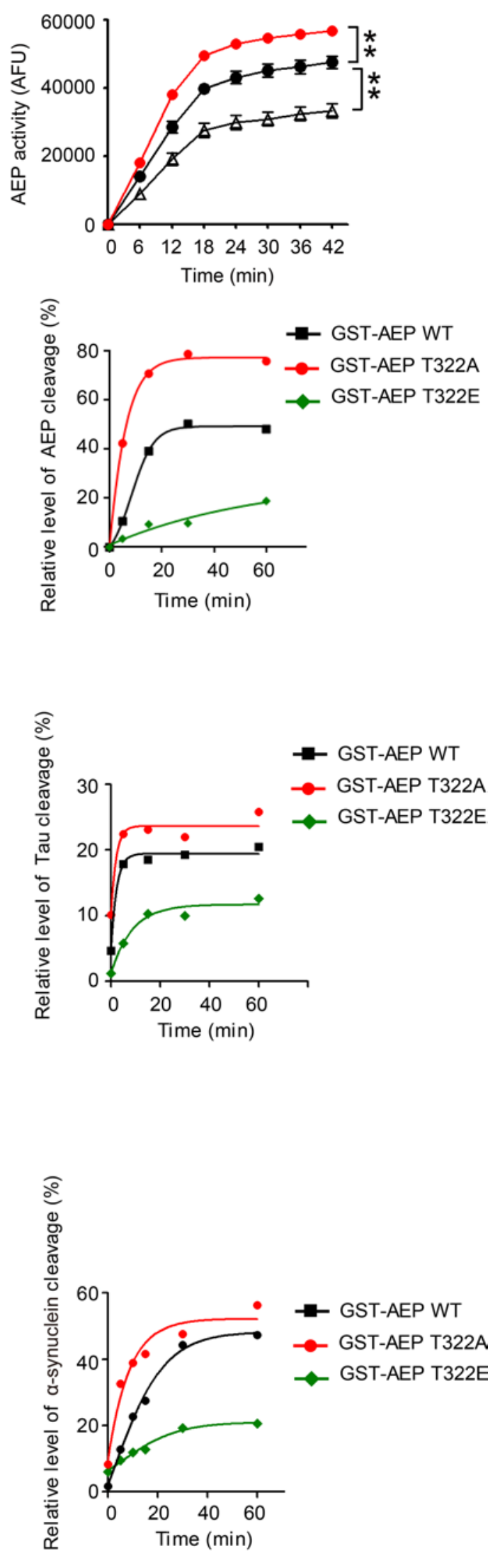
Figure 5. Akt phosphorylation of AEP inhibits its autocleavage and enzymatic activation. (A and B) AEP activity assay of HEK293 cell lysates. HEK293 cells were cotransfected with AEP and Akt WT/KD/CA (A) or WT and mutated AEP (B). Data shown as the mean \pm SEM of 3 independent experiments. ${ }^{*} P<0.05$, ${ }^{* *} P<0.01$ by 2-way ANOVA and Bonferroni's post hoc test. (C-E) Western blot analysis of autocatalytic process of WT/mutated AEP (C) and cleavage of Tau (D) and $\alpha$-synuclein (E) by WT/mutated AEP after incubation in $\mathrm{pH} 5.5$ reaction buffer at $37^{\circ} \mathrm{C}$. Truncated bands were quantified using densitometry. After being normalized with total AEP, tau, or $\alpha$-synuclein levels, the relative levels were plotted against reaction times. Each curve (right) was fitted with GraphPad Prism 5 using the Boltzmann sigmoidal function. Data are representatives of 4 independent experiments. AFU, arbitrary fluorescence units.

Similarly, Akt knockdown in BR6 or SH-SY5Y cells modified the cytotoxicity of tau P301S or GFP- $\alpha$-syn upon cleavage by AEP in the presence or absence of BDNF (Supplemental Figure 6). Hence, BDNFstimulated Akt manipulates AEP activity and its role in tau- or $\alpha$-syn-mediated cytotoxicities via T322 phosphorylation.

We also extended our investigation of the cell death effect to $\mathrm{MPP}^{+}$, a neurotoxic metabolite of MPTP. Overexpression of WT AEP induced APP N585 and tau N368 cleavage, and these effects were enhanced in the presence of T322A. Notably, $\mathrm{MPP}^{+}$treatment further elevated the proteolytic activity of AEP WT and $\mathrm{T} 322 \mathrm{~A}$, which also robustly truncated $\alpha$-syn N103. $\mathrm{MPP}^{+}$triggered weak APP, tau, and $\alpha$-syn cleavage by AEP even in the presence of T322E, suggesting that endogenous AEP was activated by $\mathrm{MPP}^{+}$in $\mathrm{BR} 6$ cells (Supplemental Figure 7A). Consequently, $\mathrm{MPP}^{+}$treatment induced much higher LDH activities in the control, which was increased by overexpression of WT AEP; cell death activity peaked with T322A, and it was significantly antagonized in the presence of T322E (Supplemental Figure 7B). To further explore BDNF-mediated Akt phosphorylation of AEP T322 in these events, we transfected BR6 cells with AEP WT and unphosphorylated T322A, followed by treatment with $\mathrm{MPP}^{+}$in the presence or absence of BDNF. Once again, both AEP WT and T322A induced tau N368 or $\alpha$-syn N103 cleavage, which was augmented by $\mathrm{MPP}^{+}$. However, only AEP WT but not T322A was responsive to BDNF, displaying reduced activities in truncating the substrates (Supplemental Figure 7C). LDH assay tightly coupled with AEP enzymatic activities (Supplemental Figure 7D). To further explore Akt-phosphorylated AEP in $\alpha$-syn-induced cell death, again, we found that the T322A mutant robustly cleaved the transfected $\alpha$-syn N103 and endogenous tau N368, inducing prominent cell death, followed by WT AEP, whereas T322E exhibited the weakest effect (Supplemental Figure 7, E and F). Therefore, BDNF via Akt phosphorylation inhibits AEP-mediated cell death, triggered by $\alpha$-syn or tau P301S.

Akt phosphorylation mimetic T322E mutant inhibits tau pathologies and ameliorates cognitive dysfunctions in Tau P301S mice. AEP was robustly truncated and activated in both fractions in Tau P301S mice but not WT control, indicating that BDNF and its downstream p-Akt signaling are repressed in Tau P301S mice, which was manifested by immunofluorescent staining of the hippocampal brain sections and BDNF ELISA (Figure 7, A-F). AEP was highly activated in Tau P301S mice and was associated with noticeable tau fragmentation (3). Hippocampal synapse loss and impaired synaptic function are detected in 3-month-old P301S mice before fibrillary tau tangles emerge (37). To explore the pathological roles of Akt-mediated AEP T322 phosphorylation in these events, we injected AAV virus expressing WT AEP or T322E mutant into the hippocampus of Tau P301S mice (3 months old). After 3 months, we examined tau cleavage by AEP and its associated pathologies. Overexpression of WT AEP elicited its autocleavage; by contrast, T322E remained intact. Consistent with these findings, human tau P301S was markedly cleaved at N368 by WT AEP, and endogenous tau N368 cleavage in Tau P301S mice was inhibited by T322E. The proteolytic cleavage effect was validated by Tau- 5 and human HT-7 tau antibodies. We made a similar observation with tau N368 truncation, with very weak activity in WT control mice (Figure 7G). AEP enzymatic assay with the hippocampal lysates confirmed that AEP was highly activated in WT but not T322E mice, with tau P301S activity stronger than in WT mice, consistent with tau N368 cleavage intensity (Figure 7H). Immunohistochemical staining revealed that both AEP WT and T322E were successfully delivered into the hippocampal CA1 region. p-Tau signals manifested by AT- 8 antibody showed that WT AEP presented the most robust effect, followed by control. In contrast, p-Tau was largely inhibited in T322E brain (Figure 7, I and J). Golgi staining indicated that the dendritic spines were significantly reduced in Tau P301S mice as compared with WT mice. Nevertheless, overexpression of T322E in Tau P301S mice markedly augmented the spines (Figure 8A). Electron microscopy analysis showed that the number of synapses in Tau P301S-Tg mice was lower than that of WT mice, which was significantly increased by T322E overexpression (Supplemental Figure 8A). 


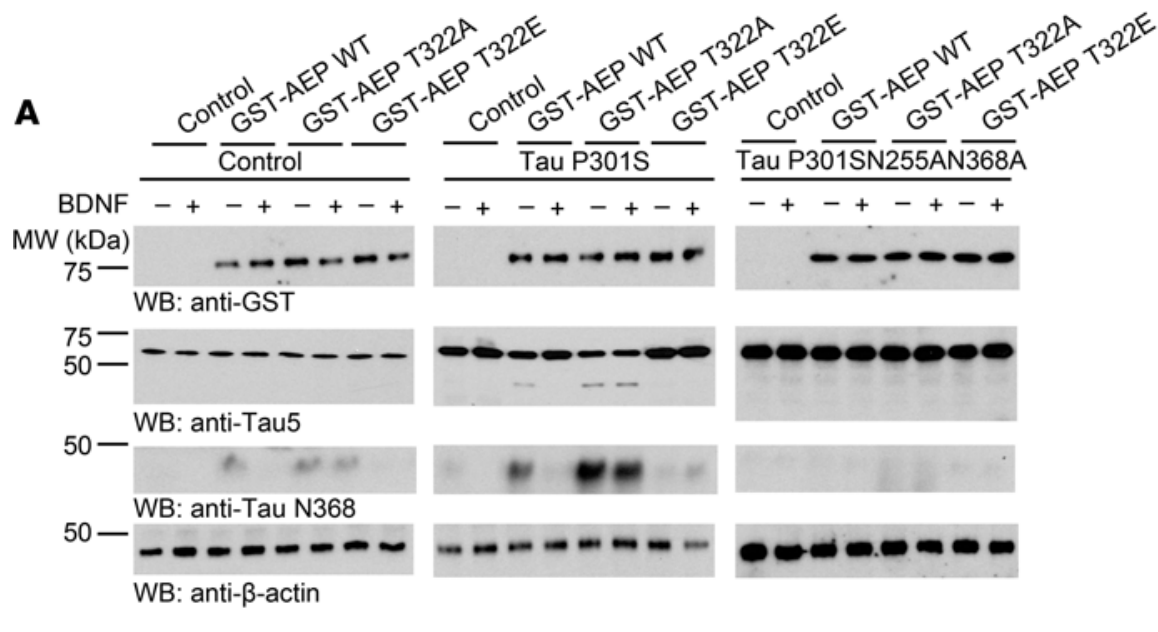

B
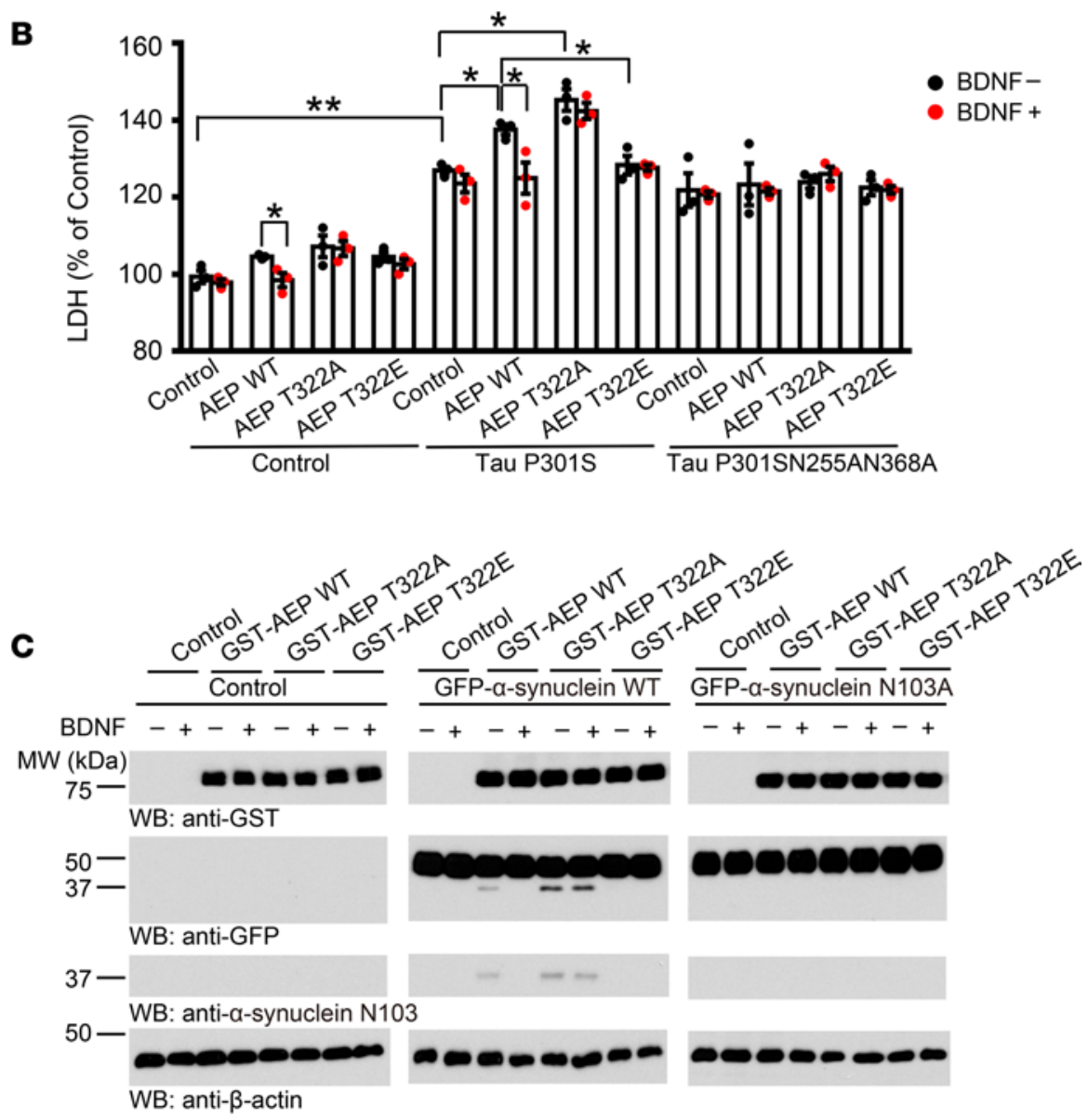

D

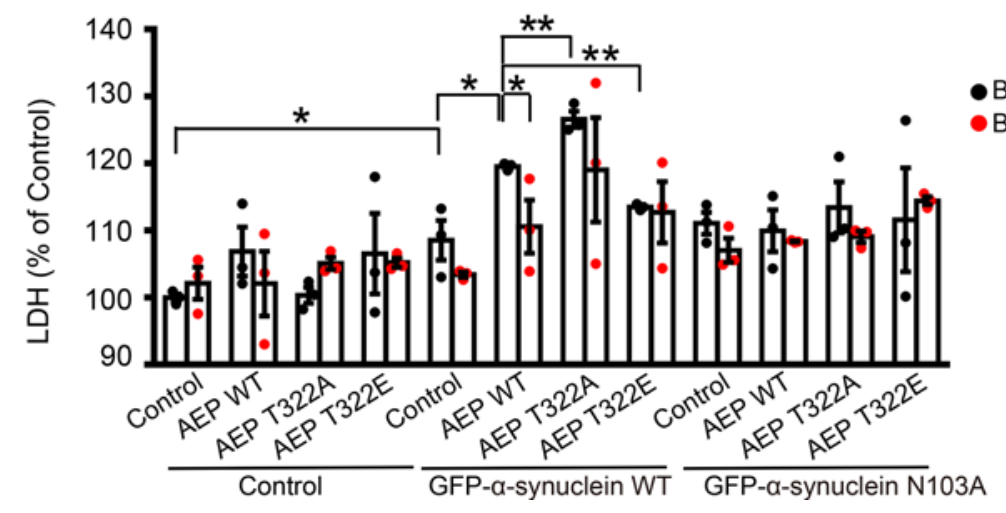

BDNF-

BDNF +
Figure 6. Alt-phosphorylated AEP blocks tau P3015 or $\alpha$-synuclein-induced cell death. (A) AEP T322A increases truncated tau and T322E mutant blocks the cleavage. Western blot was conducted using BR6 cells cotransfected with AEP and tau P301S. Cells were treated with 100 $\mathrm{ng} / \mathrm{ml}$ BDNF 1.5 hours before harvesting. (B) $\mathrm{LDH}$ assay showing that $\mathrm{AEP}$ mutants regulate cell death induced by tau P301S. Data shown as the mean \pm SEM $(n=3)$. (C) AEP T322A increases truncated $\alpha$-synuclein and T322E mutant blocks the cleavage. Western blot was conducted using BR6 cells cotransfected with AEP and $\alpha$-synuclein. Cells were treated with $100 \mathrm{ng} / \mathrm{ml}$ BDNF 1.5 hours before harvesting. (D) LDH assay showing that AEP mutants regulate cell death induced by cleaved $\alpha$-synuclein. Data shown as the mean $\pm \operatorname{SEM}(n=3) .{ }^{*} P<0.05$, ${ }^{*} P<0.01$ by 2 -way ANOVA with Bonferroni's post hoc test. 
A

Mouse brain

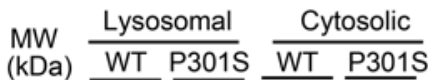

$50 \div=-\overline{-1}$

WB: anti-AEP pT322

37 - WB: anti-AEP

$100----$

$50-$ WB: anti-LAMP1
B
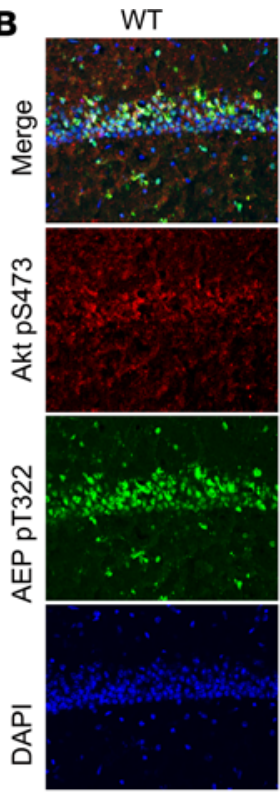
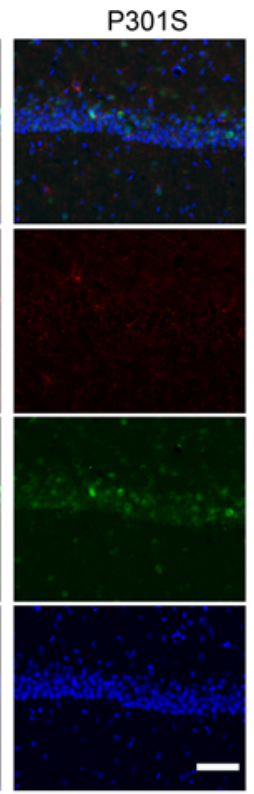

C

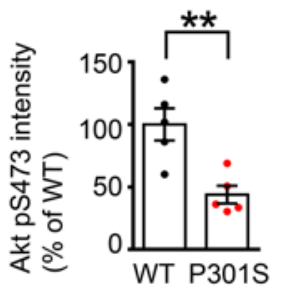

E

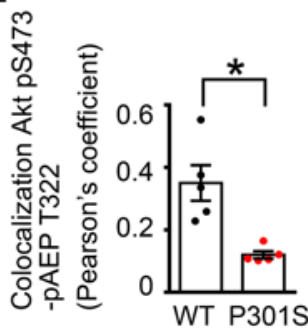

D

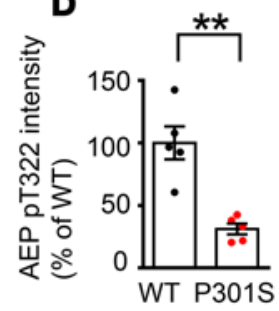

$\mathbf{F}$

BDNF ELISA

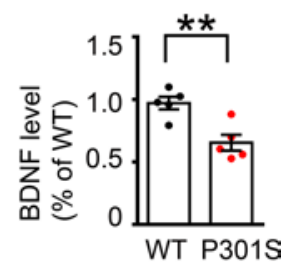

G

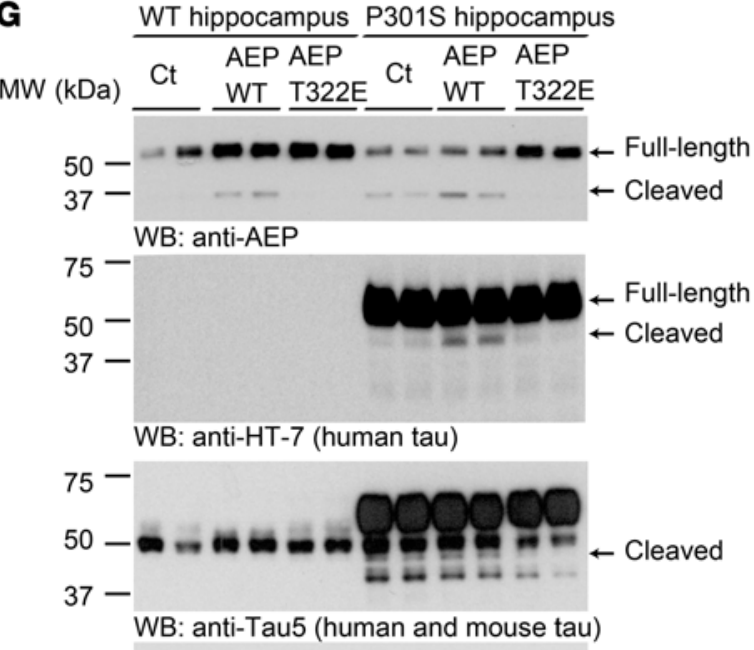

$50-$

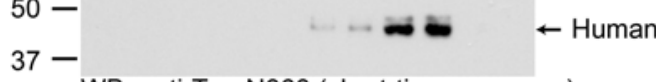

WB: anti-Tau N368 (short-time exposure)
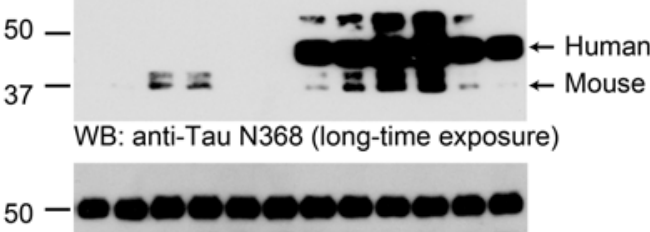

WB: anti-Tubulin

I

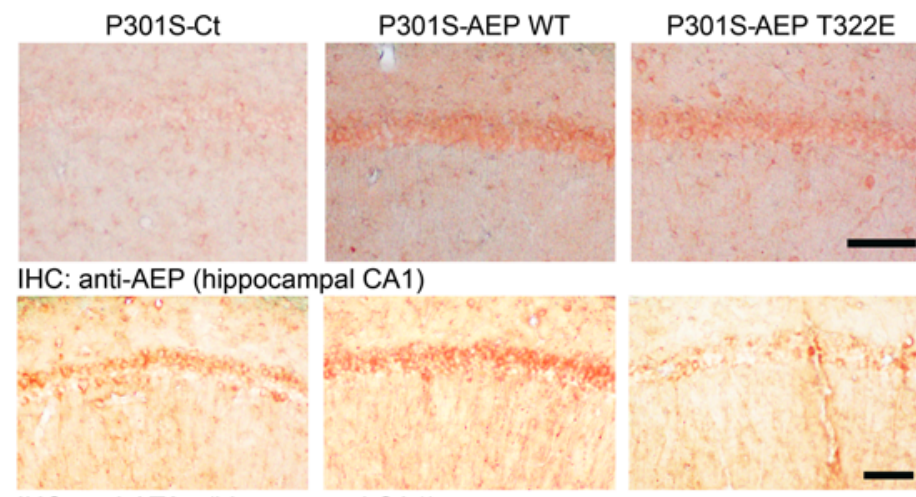

IHC: anti-AT8 (hippocampal CA1)

H

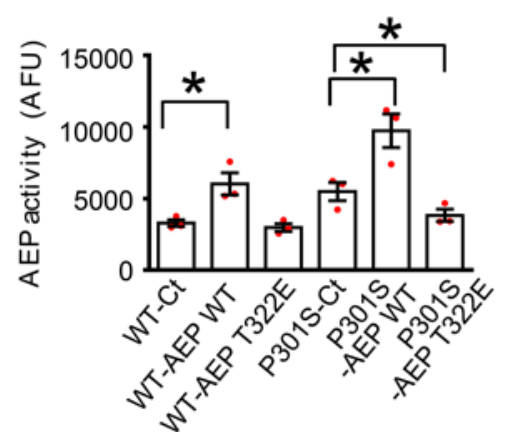

J

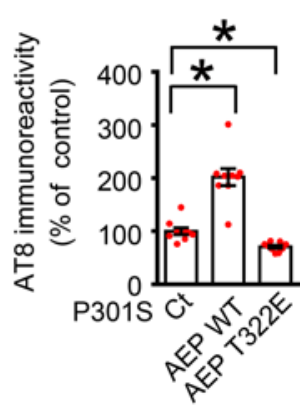

Figure 7. Akt phosphorylation mimetic T322E mutant inhibits tau pathologies in Tau P3015 mice. (A) Western blot analysis of phosphorylated AEP and Akt in 9-month-old Tau P301S mouse brains compared with age-matched WT littermates. (B-E) Immunofluorescent staining of Akt pS473 and AEP pT322 in 9-month-old Tau P3015 mouse hippocampal CA1 compared with age-matched WT littermates. Scale bar: $50 \mu \mathrm{m}$. Quantification of intensity (C and $\mathbf{D})$ and colocalization (E) are shown. Data shown as the mean \pm SEM ( $n=5$ per group). (F) Relative BDNF level by ELISA in P301S mouse hippocampus. Data shown as the mean \pm SEM ( $n=5$ per group). (G) T322E mutation reduces AEP and tau fragments. Hippocampal CA1 tissues from AAV-injected Tau P3015 mice were analyzed by immunoblotting ( $n=4$ mice per group). Molecular weight of untagged exogenous AEP is similar to endogenous AEP. (H) T322E mutation reduces AEP enzymatic activity. Data represent tyhe mean \pm SEM ( $n=3$ mice per group). (I and J) Tau pathology is attenuated by AEP T322E. IHC staining with anti-p-Tau AT8 antibody. The brain sections were immunostained with AEP (upper) and AT8 (middle). Scale bar: $25 \mu \mathrm{m}$. AT- 8 immunoreactivity quantification (lower, mean \pm SEM; 9 sections from 3 mice). Western blot data in $\mathbf{A}$ and $\mathbf{G}$ are representative of 3 independent experiments. ${ }^{*} P<0.05 ;{ }^{* *} P<0.01$ by 2 -tailed $t$ test $(\mathbf{C}-\mathbf{F})$ or 1 -way ANOVA with Tukey's multiple-comparisons test (H and $\left.\mathbf{J}\right)$. 
Electrophysiological analysis found that the paired-pulse ratios were the lowest in Tau P301S WT AEP mice, and T322E substantially enhanced the signals. In contrast, there was no major difference among control, AEP WT and T322E in WT mice. The LTP in the hippocampal CA1 region that represents the molecular basis of learning and memory was diminished in Tau P301S-Tg mice compared with WT mice. T322E overexpression profoundly rescued the impaired synaptic function (Figure 8B). Hence, AEP phosphorylation on T322 by Akt greatly alleviates the synaptic deficiency in Tau P301S mice. In agreement with the synaptic plasticity, cognitive behavioral tests with the Morris water maze demonstrated that Tau P301S mice displayed noticeable learning and memory deficits compared with WT mice, which were highly restored by T322E overexpression (Figure 8, C-E). The distance to the mount platform was consistent with the latency to the submerged platform. The swim speed remained comparable among the groups (Supplemental Figure 8, B-D). Next, we performed fear conditioning tests. AEP T322E increased the memory in both the cued fear test and the contextual fear assay relative to control in Tau P301S mice (Supplemental Figure 8, E and F). Therefore, Akt phosphorylation of AEP prevents tau N368 cleavage, rescuing the synaptic dysfunctions and cognitive deficits in Tau P301S mice.

Akt-unphosphorylated AEP T322A mutant facilitates $\alpha$-syn pathologies and stimulates motor deficits in young $\alpha$-SNCA mice. $\alpha$-SNCA-Tg mice display extensive tyrosine hydroxylase (TH) loss and motor defects at 12 to 18 months of age (38). To assess whether the Akt-unphosphorylated AEP T322A mutant that possesses increased enzymatic activity facilitates $\alpha$-syn cleavage and PD-like pathologies in young $\alpha$-SNCA mice, we administered adeno-associated virus (AAV) expressing control, WT AEP, or AEP T322A into the SN of 2-month-old $\alpha$-SNCA mice. We monitored dopaminergic neuron loss 2 months after viral injection. Immunofluorescent staining with anti-TH showed that overexpression of WT AEP evidently decreased TH numbers in both the SN and striatal regions (Figure 9A), which was further elevated by the T322A mutant, consistent with the observation that T322A strongly cleaves $\alpha$-syn and promotes dopaminergic cell death (Figures 5E and 6C). Fluorescence intensity analysis validated these observations (Figure 9, B and C). To investigate the effect of $\mathrm{T} 322 \mathrm{~A}$ on motor functions, we conducted a panel of behavioral tests including the Rotarod test, cylinder test, and amphetamine-induced rotation. T322A-administered mice exhibited much less latency than WT AEP mice, followed by the control in the Rotarod assay, consistent with abundant $\alpha$-syn N103 and the most severe TH loss (Figure 9D). We made similar observations in the cylinder test (Figure 9E). In alignment with these findings, T322A overexpression also triggered highly increased amphetamine-induced rotational asymmetry, as compared with WT AEP mice that exhibited much stronger rotational activity than control (Figure 9F). Immunoblotting showed that AEP T322A was more highly activated than WT AEP and control; accordingly, $\alpha$-syn N103 was substantially produced, with T322A much stronger than WT. By contrast, no $\alpha$-syn N103 was detected in AAV control-injected samples (Figure 9G). In accordance with these observations, immunofluorescence costaining showed that TH-positive dopaminergic neurons were barely detectable in WT AEP- and T322A-injected SN. Consequently, evident $\alpha$-syn N103 was identified (Supplemental Figure 9A). AEP enzymatic activity mirrored $\alpha$-syn N103 intensities in the brain samples (Figure 9H). LBs are composed of highly phosphorylated $\alpha$-syn and numerous other components including 14-3-3. Correspondingly, we performed costaining with both $\alpha$-syn and 14-3-3 antibodies in the SN region of the brain sections. In T322A mice, aggregated $\alpha$-syn colocalized with 14-3-3 or ubiquitin in the remnant dopaminergic neurons (Figure 9I). Immunofluorescence costaining with $\mathrm{TH}$ and p- $\alpha$-syn (S129) antibodies showed that in the remnant TH-positive dopaminergic neurons, there was no detectable $\mathrm{p}$ - $\alpha$-syn signal in control, whereas prominent $\mathrm{p}$ - $\alpha$-syn staining was found in both WT AEP- and T322A-infected mice (Supplemental Figure 9B). Together, these behavioral assays support the hypothesis that Akt-unphosphorylated AEP T322A mutant elicits more severe motor impairment than WT AEP and control in young $\alpha$-SNCA mice, coupling with robust $\alpha$-syn N103 cleavage and extensive TH loss.

\section{Discussion}

In the current study, we found that AEP is phosphorylated by Akt on residue T322 upon BDNF treatment and translocates into the lysosome, leading to inactivation of the enzyme. When BDNF is reduced in neurodegenerative diseases, AEP T322 phosphorylation is attenuated and the enzyme leaks from lysosomes into the cytoplasm, where it is activated and cleaves numerous key pathological players implicated in various neurodegenerative diseases. Mounting evidence supports the notion that BDNF/TrkB signaling is implicated in the pathogenesis of neurodegenerative diseases including $\mathrm{AD}$ and PD. For instance, BDNF and its receptor TrkB are reduced in brains of mild cognitive impairment (MCI) or early AD patients (39-42) and 
A
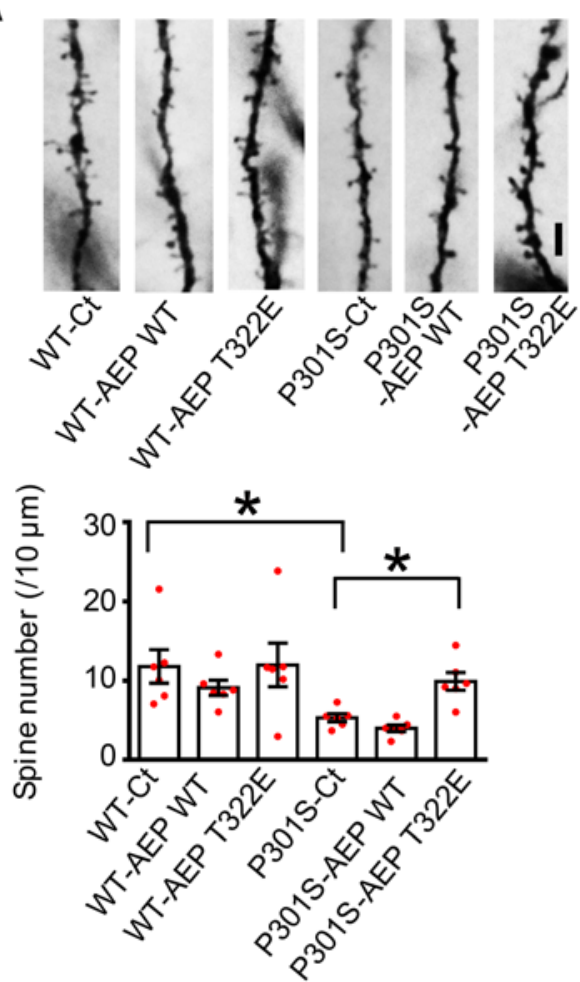

C

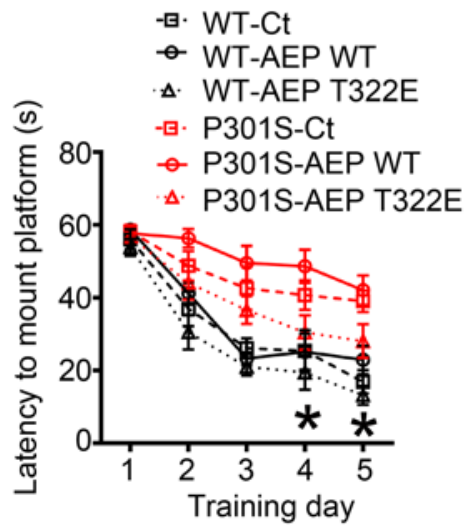

B

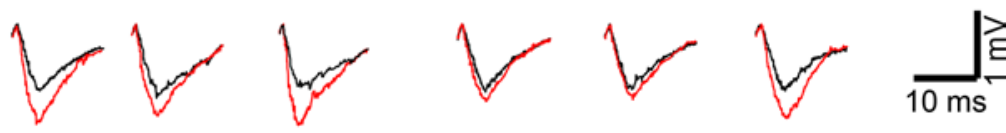

WT- WT- WT- P301S- P301S- P301S-

Ct AEP WT AEP T322E Ct AEP WT AEP T322E
口WT-Ct
OWT-AEP WT
$\square$ P301S-Ct
O P301S-AEP WT

$\triangle$ WT-AEP T322E

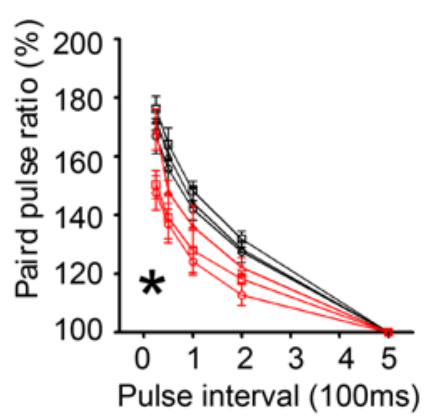

$\triangle$ P301S-AEP T322E

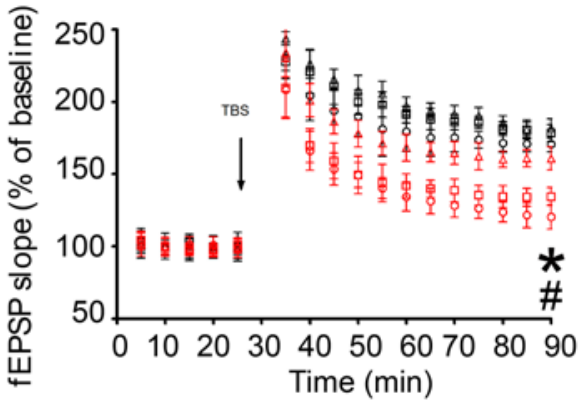

D

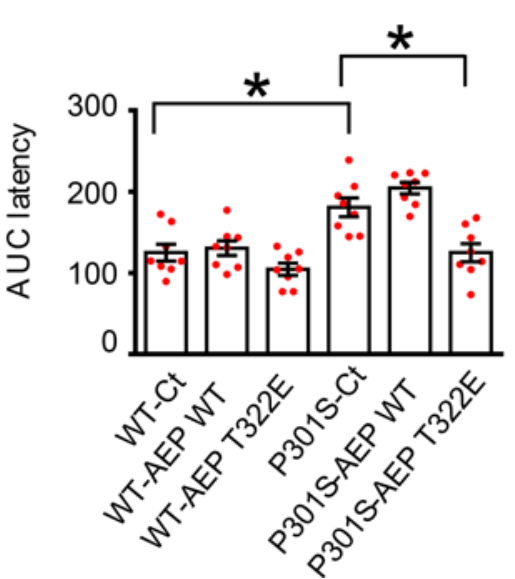

$\mathbf{E}$

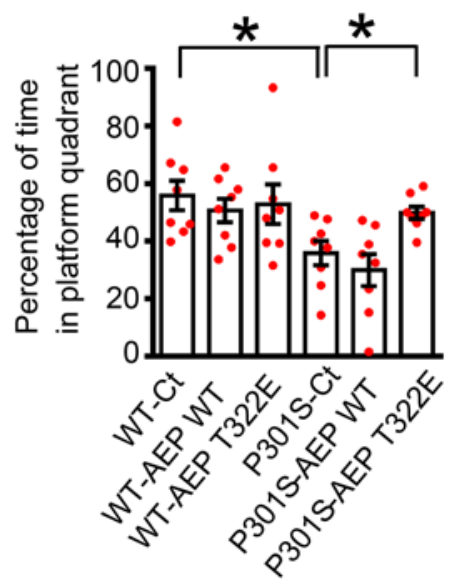

Figure 8. Akt phosphorylation mimetic T322E mutant ameliorates cognitive dysfunctions in Tau P3015 mice. (A) T322E mutant increases the spine density. Scale bar: $5 \mu \mathrm{m}$. Golgi staining was conducted on brain sections from CA1 regions of Tau P301S mice (mean \pm SEM; $n=6$ ). ${ }^{*} P<0.05$ by 1 -way ANOVA with Tukey's multiple-comparisons test. (B) Electrophysiological analysis. Shown traces are the representative fEPSPs recorded before (black) and 60 minutes after (red) 3-theta-burst stimulation. T322E mutant increased the ratio of paired pulses (lower left) and rescued the LTP defects in Tau P301S mice (lower right) (mean \pm SEM; $n=6$ in each group). ${ }^{*} P<0.05$ P301S-T322E versus P301S-Ct; ${ }^{*} P<0.05$ P301S-T322E versus P301S-AEP WT; 1-way ANOVA with Tukey's multiple-comparisons test. (C-E) Morris water maze analysis of cognitive functions. T322E mutant in CA1 rescues the learning (C and $\mathbf{D})$ and memory (E) impairments in Tau P3015 mice (mean \pm SEM; $n=8$ mice per group). ${ }^{*} P<0.05$ by 2-way ANOVA with Bonferroni's post hoc test for $\mathbf{C}$ or 1-way ANOVA with Tukey's multiple-comparisons test for $\mathbf{D}$ and $\mathbf{E}$.

postmortem PD patients' SN $(43,44)$, raising the possibility that there may be a link between BDNF and $\mathrm{AD}$ and PD. Moreover, various BDNF polymorphisms, in particular V66M and C270T, are associated with increased risk of developing $\mathrm{AD}$ and $\mathrm{PD}$ (45-49). Further, central BDNF administration or gene delivery into rodent and primate models of $\mathrm{AD}$ produces beneficial effects against $\mathrm{A} \beta$-induced neurotoxicity, synapse loss, and impairments in learning and memory $(27,28)$. BDNF also protects dopaminergic neurons from loss and motor defects induced by 6-OHDA or $\operatorname{MPTP}(30,31,50)$. Intriguingly, pathogenic $\alpha$-syn mutation 
A
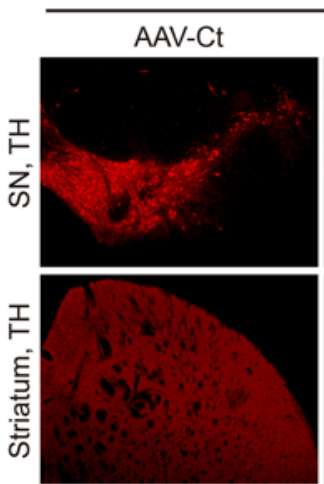

D

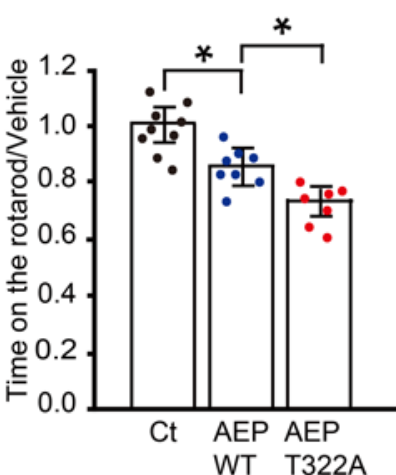

H

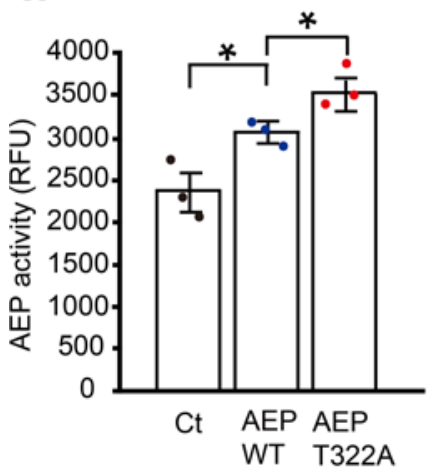

SNCA TG
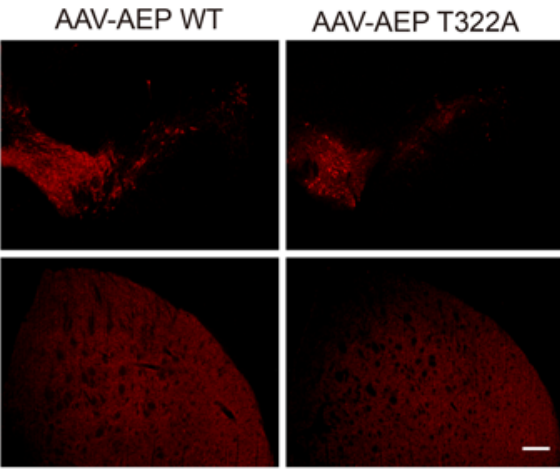

B

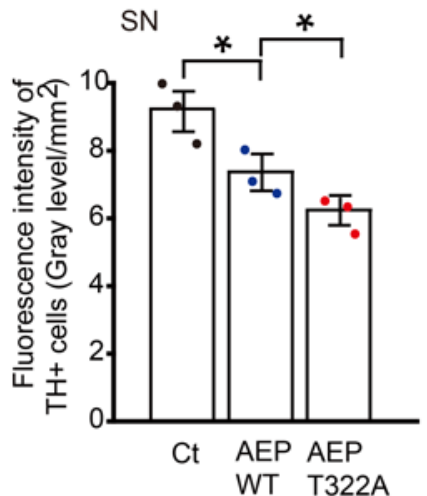

C

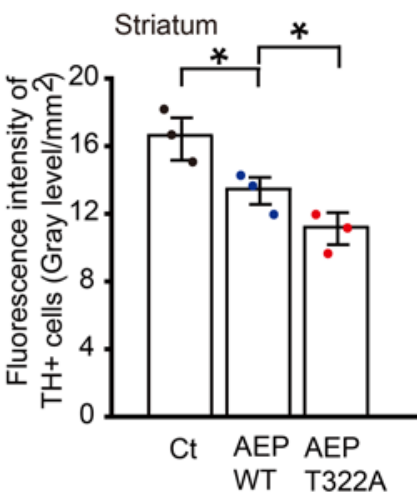

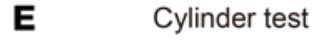

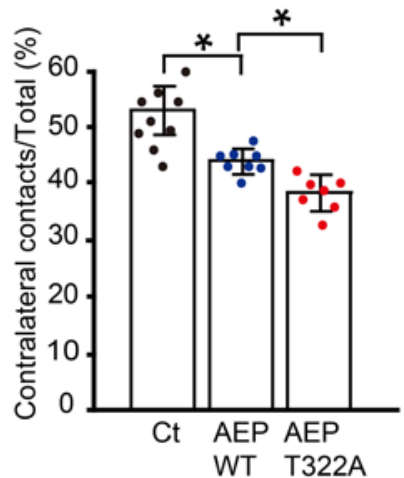

F Amphetamine-induced rotation
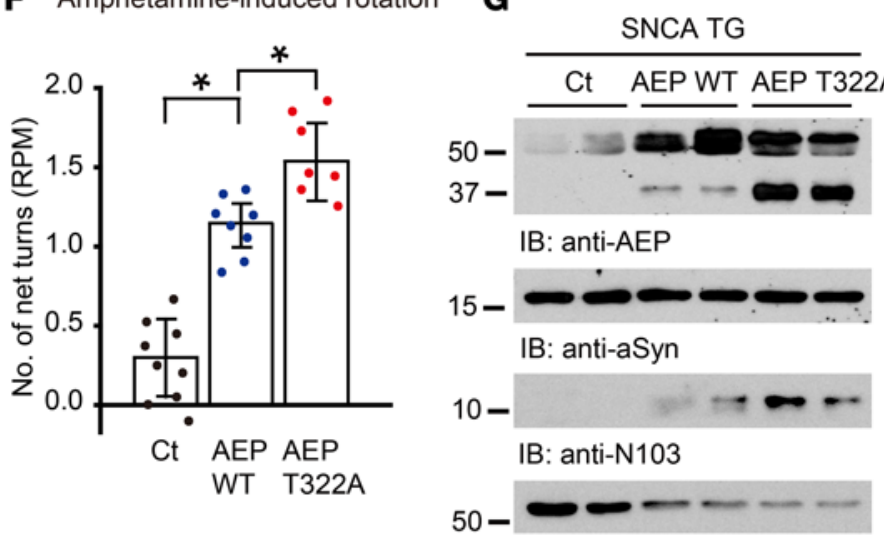

IB: anti-TH

I
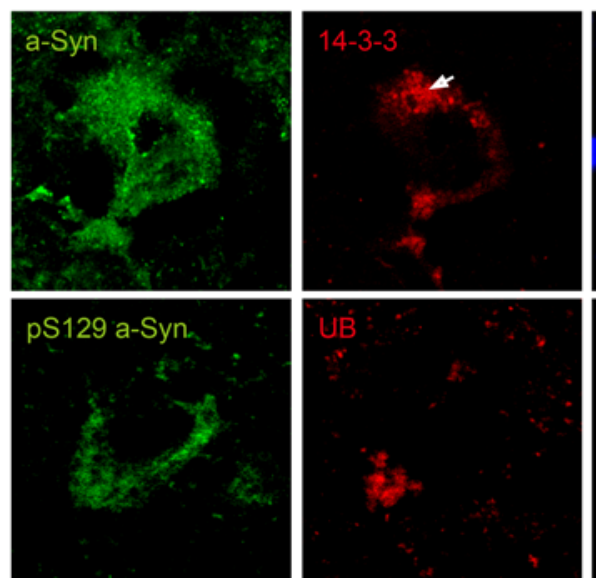
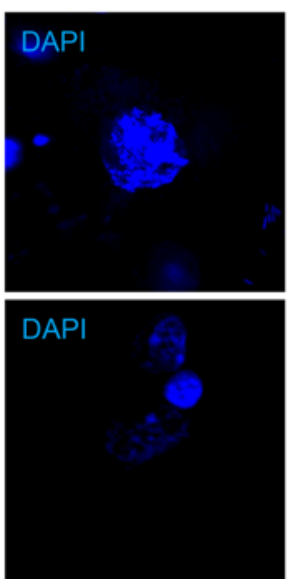
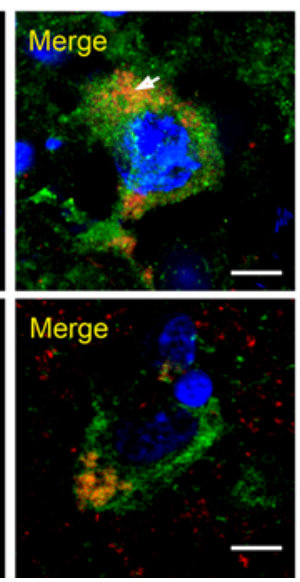

Figure 9. Unphosphorylated AEP T322A mutant facilitates $\alpha$-synuclein pathologies and stimulates motor deficits in young SNCA-Tg mice. (A-F) AEP T322A overexpression induces more $\mathrm{TH}^{+}$dopaminergic cell loss and motor dysfunction in SNCA mice, compared with control or AEP-overexpressing group. AAV-control (Ct), AAV-AEP, or AAV-AEP T322A was injected into right substantial nigra (SN) of SNCA-Tg mice. (A) TH expression in $\mathrm{SN}$ and striatum of the above animals was analyzed by immunofluorescent staining. Scale bar: $200 \mu \mathrm{m}$. (B and C) Quantification of TH+ fluorescent signals in SN (B) and striatum (C). Data are shown as the mean \pm SEM ( $n=3$ per group). Motor behavioral assays, Rotarod (D), cylinder test (E), and amphetamine-induced rotation (F) were conducted by a blinded observer 2 months after the virus injection. AAV-AEP T322A infection induced more severe motor dysfunction than AAV-AEP WT. Data are shown as the mean \pm SEM ( $n=7-9$ per group). (G) The T322A mutation enhances AEP's protease activity and cleavage of $\alpha$-synuclein. SN lysates were probed with various indicated antibodies. (H) AEP enzymatic assay. AEP activity in AEP T322A-injected mice was increased more than in the AEP WT-injected group. Data are shown as mean \pm SEM ( $n=3$ per group). (I) $\alpha$-Synuclein localizes in the Lewy body in the SN of AEP T322A-injected brains. Immunofluorescent signals of anti- $\alpha$-synuclein (green) and anti-14-3-3 (red) or anti- $\alpha$-synuclein pS129 (green) and anti-ubiquitin (red) as Lewy body marker were detected in AEP T322A-injected brain sections. The nuclei were stained with DAPI. Scale bar: $20 \mu \mathrm{m} .{ }^{*} P<0.05$ by 1 -way ANOVA. 
(A30P or A53T) also links to BDNF production suppression (29). Nonetheless, recent studies indicate that $\mathrm{BDNF}$ reduction in $3 \times \mathrm{Tg}\left(3 \times \mathrm{Tg} / \mathrm{BDNF}^{+/}\right)$mice does not alter $\mathrm{A} \beta$ or tau pathology (51). Additionally, TrkB reduction in $5 \times \mathrm{FAD}$ mice does not affect $\beta$-amyloidogenesis, although it exacerbates spacial memory deficits (52). Hence, it has been suggested that BDNF reduction may not contribute to AD or PD pathogenesis. However, in these studies, BDNF or TrkB is partially eliminated, and neurotrophic signaling remains intact, which may account for the failure to observe changes in $\mathrm{A} \beta$ or tau pathologies. On the other hand, because both $\mathrm{A} \beta$ and tau pathologies are overwhelmed in these $\mathrm{AD}$ mouse models, the high baselines might block the pathological effects induced by BDNF depletion. Here, we provide evidence demonstrating that $\mathrm{BDNF} /$ TrkB neurotrophic signaling reduction leads to a decrease in Akt activity, resulting in AEP T322 phosphorylation attenuation and proteolytic activation elevation. BDNF triggers AEP T322 phosphorylation and inhibits its protease activity, decreasing $A \beta$ production. Consistently with this notion, BDNF reduces $A \beta$ levels in vitro and in vivo and BDNF deprivation elevates $A \beta$ production $(27,53,54)$. Furthermore, BDNF dampens tau N368 cleavage by AEP. This finding is in alignment with a previous report that BDNF rapidly induces tau dephosphorylation via TrkB signaling (55).

Neurodegenerative diseases are progressive in neuronal loss, with age being the major risk factor. Our recent evidence strikingly supports that AEP is a predominant age-dependent protease in AD and PD pathology progression (2-4). In addition, we recently reported that the cell-cycle kinase SRPK2 phosphorylates human AEP on S226 and triggers its activation (14). Accumulating evidence demonstrates that postmitotic neurons reenter the cell cycle but are incapable of accomplishing cell division. Degenerative neurons reinitiate the cell cycle by expressing cyclins and Cdks. Instead of going into cell division, these neurons go into apoptosis (56). We have shown that SRPK2 is highly activated in neurodegenerative diseases, coupling cell cycle and cell death (57). Here, we provide an additional layer of molecular regulation of AEP by BDNF-activated Akt. Conceivably, deficient BDNF in neurodegenerative diseases may activate AEP, and combine with SRPK2 phosphorylation of AEP S226. These 2 pathways might additively orchestrate the elevation of AEP activity, leading to extensive APP, tau, and $\alpha$-syn fragmentation and aggregation.

Pro-AEP is bimodular: at higher $\mathrm{pH}$, the $\mathrm{C}$-terminal cap domain covers the core domain and sterically obstructs access to the active site, whereas at a lower $\mathrm{pH}$, the cap domain is removed through autoproteolysis. The autocleavage of AEP occurs at residue N323. Removal of the cap domain enables substrate access to the catalytic dyad of $\mathrm{H} 150$ and $\mathrm{C} 191$ for the subsequent catalytic cleavage of the peptide substrate $(58,59)$. Noticeably, Akt phosphorylates residue T322, which is right next to the cleavage-site asparagine. Presumably, Akt phosphorylates T322 by covalently adding a negatively charged $\left(\mathrm{PO}_{4}\right)^{3-}$ group on threonine, preventing the cysteine proteinase from attacking the asparagine. BDNF and TrkB are expressed in both cholinergic and dopaminergic neurons and play critical roles in promoting neuronal survival. BDNF exerts impressive therapeutic efficacy toward $\mathrm{AD}$ and $\mathrm{PD}$ in numerous animal models, justifying that this pathway via Akt plays an essential role in dictating AEP activation status. T322 phosphorylation leads to the translocation of $\mathrm{p}$-AEP into the lysosomes, sequestering AEP from caspase-mediated truncation. It has been reported previously that other proteases including caspases, thrombin, and calpains may cut tau or $\alpha$-syn (60). However, none of these proteases is upregulated and activated in an age-dependent manner and simultaneously cleave both tau and $\alpha$-syn in AD and PD. Thus, AEP might be a crucial and unique drug target for developing disease-modifying therapies.

Together, employing gain-of-function and loss-of-function with unphosphorylated T322A and phosphorylation mimetic T322E mutants, we provide convincing evidence supporting the idea that BDNF via its downstream effector Akt phosphorylation regulates the catalytic activation of the crucial age-dependent protease AEP. Reduction of BDNF and its receptor TrkB in neurodegenerative diseases may induce AEP activation, implicating them in the onset of pathogenesis and progression of neurodegenerative diseases.

\section{Methods}

Antibodies and reagents. Anti-p-Akt substrate, Cell Signaling Technology, catalog 9611s; anti-GST (peroxidase conjugate), Sigma-Aldrich, catalog A7340; anti-HA, Santa Cruz Biotechnology, catalog sc-7392; antiGFP, Santa Cruz Biotechnology, catalog sc-9996; anti-Akt, Cell Signaling Technology, catalog 4691S; antiAkt pS473 rabbit mAb, Cell Signaling Technology, catalog 4060; anti-p-Akt (Ser473) antibody, clone 6F5, EMD Millipore, catalog 05-1003; anti-Akt pT308, Cell Signaling Technology, catalog 13038S; anti-AEP 6E3, a gift from Dr. Colin Watts, Professor of Immunobiology, Division of Cell Signalling and Immunology, College of Life Sciences, University of Dundee, Dundee, UK; anti-AEP pT322, Ye Lab; anti-tau 
N368, Ye Lab; anti-APP N585, Ye Lab; anti- $\alpha$-syn N103, Ye Lab; anti- $\alpha$-tubulin,Sigma-Aldrich, T6074; anti- $\beta$-actin, Abcam, ab8227; anti-LAMP1, Santa Cruz Biotechnology, catalog sc-5570; anti-LAMP1, Santa Cruz Biotechnology, catalog sc-17768; anti-acinus pS422, EMD Millipore, catalog 07-1840; anti-GST (Alexa Fluor 488 conjugate), Cell Signaling Technology, catalog 3368; anti-p-tau Ser202, Thr205 (AT8), Thermo Fisher Scientific, catalog MN1020; anti- $\alpha$-syn, Santa Cruz Biotechnology, catalog sc-69977; anti- $\alpha$-syn pS129, Santa Cruz Biotechnology, catalog sc-135638; anti-TrkB, R\&D Systems, MAB397; antiTrkB pY706, Santa Cruz Biotechnology, catalog sc-135645; anti-TrkB pY816, Ye Lab; anti-BDNF, Novus, NBP2-42215; Glutathione Sepharose 4B, GE Healthcare, catalog 17075601; ATP, $\left[\gamma^{-32} \mathrm{P}\right]$, PerkinElmer, catalog BLU002250UC; Protein A/G PLUS-Agarose, Santa Cruz Biotechnology, catalog sc-200, rBDNF, Peprotech, catalog 450-02; DAPI, Sigma-Aldrich, catalog D9542; Streptavidin, Alexa Fluor 594 conjugate, Thermo Fisher Scientific, catalog S11227; AEP substrate Z-Ala-Ala-Asn-AMC, Bachem; silver nitrate, Sigma-Aldrich, catalog 209139; AEP p-T322 peptide antigen, Covance; Akt1/2 I, Sigma-Aldrich, catalog A6730; wortmannin, EMD Millipore, catalog 681675; K252a, EMD Millipore, catalog 420298; LY294002, Sigma-Aldrich, catalog L9908; PD98059, Sigma-Aldrich, catalog P215; QuikChange Lightning SiteDirected Mutagenesis Kit, Agilent Technologies, catalog 210518; EZ-Link Sulfo-NHS-LC-Biotinylation Kit, Thermo Fisher Scientific, catalog 21435; Histostain-SP IHC Kit, mouse, Thermo Fisher Scientific, catalog 956543B; CytoTox 96 Non-Radioactive Cytotoxicity Assay, Promega, catalog G1780.

Mice, primary cultured rat neurons, cell lines, and human tissue samples. WT C57BL/6J mice, Tau P301S mice, and human SNCA-Tg mice were purchased from the Jackson Laboratory (000664, 008169, and 023837). The $\mathrm{Lgmn}^{-/-}$mice on a mixed 129/Ola and C57BL/6 background were generated as previously reported (11). Primary cortical neurons were cultured as previously described (3). All rats were purchased from the Jackson Laboratory. HEK293 cells were cultured in high-glucose DMEM supplemented with 10\% fetal bovine serum (FBS), penicillin (100 units $/ \mathrm{ml})$, and streptomycin $(100 \mu \mathrm{g} / \mathrm{ml})$ (all from Hyclone). SHSY5Y Trk-NULL and SH-SY5Y (TrkB, BR6) cells were cultured in DMEM/F12 supplemented with 10\% FBS, penicillin (100 units $/ \mathrm{ml})$, and streptomycin $(100 \mu \mathrm{g} / \mathrm{ml})$ (all from Hyclone). Cells were incubated at $37^{\circ} \mathrm{C}$ in a humidified atmosphere of $5 \% \mathrm{CO}_{2}$. Postmortem brain samples were dissected from frozen brains of $\mathrm{AD}, \mathrm{PD}, \mathrm{DLB}$, and aged-match nondemented controls from the Emory Alzheimer's Disease Research Center. The study was approved by the Biospecimen Committee of the Emory University School of Medicine. AD was diagnosed according to the criteria of the Consortium to Establish a Registry for AD and the National Institute on Aging. Diagnoses were confirmed by the presence of amyloid plaques and NFTs in formalin-fixed tissue. Informed consent was obtained from the subjects. PD and LBD cases were clinically diagnosed and neuropathologically confirmed.

Transfection and infection of the cells. HEK293 transfection was performed using Lipofectamine 2000 (Invitrogen). SH-SY5Y and BR6 transfection was performed using Lipofectamine 3000 (Invitrogen). AAVcontrol and AAV-AEP WT/T322A/T322E used in vivo were packaged by the Manfredsson laboratory at Michigan State University. Viral titer was $1 \times 10^{13}$ to $5 \times 10^{13} \mathrm{GC} / \mathrm{ml}$. AAV-GFP and AAV-Cre-GFP were from Vector Biolabs (catalog 7019) with a titer of $1 \times 10^{13} \mathrm{GC} / \mathrm{ml}$.

In vitro kinase assay. Human recombinant GST, tau, and AEP WT/T322A proteins were expressed in HEK293 cells and purified using Glutathione Sepharose 4B Beads or by immunoprecipitation. Recombinant mouse His-tagged AEP proteins were purchased from Sino Biological (50051-M07H). Recombinant human GST-tagged Akt proteins were purchased from Cell Sciences (CRA004A). Substrates were incubated with Akt in a kinase reaction buffer $(25 \mathrm{mM}$ Tris- $\mathrm{HCl}$, $\mathrm{pH} 7.5$, with $10 \mathrm{mM} \mathrm{MgCl}, 2 \mathrm{mM}$ DTT, $5 \mathrm{mM}$ $\beta$-glycerophosphate, $0.1 \mathrm{mM} \mathrm{Na}_{3} \mathrm{VO}_{4}$, and $0.1 \mathrm{mg} / \mathrm{ml}$ heparin) containing $20 \mu \mathrm{M}$ ATP and $1.5 \mu \mathrm{Ci}$ of $\left[\gamma_{-}{ }^{32} \mathrm{P}\right]$ ATP for 2 hours at $30^{\circ} \mathrm{C}$. Reactions were then separated in SDS-PAGE and analyzed by autoradiography.

Identification of phosphorylation sites on AEP by liquid chromatography-tandem mass spectrometry. Identification of phosphorylation sites on GST-AEP in HEK293 cells cotransfected with HA-Akt was performed based on an optimized LC-MS/MS system (61). The GST-AEP band was cut into approximately $1-\mathrm{mm}^{3}$ pieces followed by in-gel digestion and peptide extraction. The resulting peptides were analyzed by nanoscale reverse-phase LC coupled with an LTQ Orbitrap Elite mass spectrometer (Thermo Fisher Scientific). The digests were dissolved in 5\% formic acid, loaded onto a $75-\mu \mathrm{m}$ inner diameter $\times 40$-cm column with 1.9- $\mu \mathrm{m} \mathrm{C18}$ resin (Dr. Maisch HPLC GmbH, Germany) and then eluted during a 95-minute gradient of $15 \%-50 \%$ B (solvent A: $0.1 \%$ formic acid; solvent B: $0.1 \%$ formic acid, $70 \%$ acetonitrile; flow rate of 260 $\mathrm{nl} / \mathrm{min}$ ). The mass spectrometer was operated in data-dependent mode with MS1 survey scans in the Orbitrap ( $1 \times 10^{6}$ target value; 240,000 resolution; $300-1,400 \mathrm{Th}, 2.0 \mathrm{Th}$ isolation window) followed by MS/MS 
scans analyzed in the ion trap (top 16, CID fragmentation; normalized collision energy of $35 ; 2 \times 10^{4}$ target value). Dynamic exclusion duration was set to 15 seconds. Maximum injection times were set to $50 \mathrm{~ms}$ for all MS1 scans and $100 \mathrm{~ms}$ for MS/MS scans. Acquired MS/MS raw files were converted into mzXML format and searched by JUMP algorithm against a composite target/decoy database to estimate false discovery rate (FDR) (62). The target protein database was downloaded from the Uniprot rat database $(28,863$ protein entries) and the decoy protein database was generated by reversing all target protein sequences.

Generation of antibodies specific for Akt-phosphorylated AEP (anti-AEP p T322). Two rabbits were immunized with the peptide Ac-CRKLMN(pT)NDLEES-amide. The rabbits received booster injections 4 times with the immunizing peptide with 3-week intervals between injections. The antiserum was affinity purified by chromatography with the immunizing peptide and was then adsorbed to a peptide spanning the phosphorylated site (Ac-CRKLMNTNDLEES-amide).

Subcellular fractionation. The subcellular fraction protocol was as previously described (8).

AEP activity assay. Tissue homogenates or cell lysates $(10 \mu \mathrm{g})$ were incubated in $200 \mu \mathrm{l}$ reaction buffer (20 mM citric acid, $60 \mathrm{mM} \mathrm{Na}_{2} \mathrm{HPO}_{4}, 1 \mathrm{mM}$ EDTA, 0.1\% CHAPS, and $1 \mathrm{mM}$ DTT, pH 5.5) containing 20 $\mu \mathrm{M}$ AEP substrate Z-Ala-Ala-Asn-AMC (Bachem). AMC released by substrate cleavage was quantified by measuring at $460 \mathrm{~nm}$ in a fluorescence plate reader at $37^{\circ} \mathrm{C}$ in kinetic mode.

AEP cleavage assay. HEK293 cells were transfected with GST-AEP WT/T322A/T322E or cotransfected with GST-tau or $\alpha$-syn. Forty-eight hours after transfection, the cells were lysed in buffer (50 $\mathrm{mM}$ sodium citrate, $5 \mathrm{mM}$ DTT, $0.1 \%$ CHAPS, 0.5\% Triton X-100, $1 \mathrm{mM}$ EDTA, and $60 \mathrm{mM} \mathrm{Na}_{2} \mathrm{HPO}_{4}$, $\mathrm{pH}$ 5.5) and centrifuged for 15 minutes at $14,000 \mathrm{~g}$ at $4^{\circ} \mathrm{C}$. The supernatants were then incubated at $37^{\circ} \mathrm{C}$ for $0,5,10,15,30$, and 60 minutes. The samples were then boiled in $1 \times$ SDS loading buffer and analyzed by immunoblotting.

Immunoprecipitation and Western blot analysis. Cells were washed with ice-cold PBS and lysed in coimmuprecipitation buffer (50 mM Tris- $\mathrm{HCl}$ [pH 7.5], $150 \mathrm{mM} \mathrm{NaCl}, 1 \%$ Nonidet P-40, $5 \mathrm{mM}$ EDTA, $5 \mathrm{mM}$ EGTA, $15 \mathrm{mM} \mathrm{MgCl}_{2}, 60 \mathrm{mM} \beta$-glycerophosphate, $0.1 \mathrm{mM}$ sodium orthovanadate, $0.1 \mathrm{mM} \mathrm{NaF}, 0.1$ $\mathrm{mM}$ benzamide, $10 \mu \mathrm{g} / \mathrm{ml}$ aprotinin, $10 \mu \mathrm{g} / \mathrm{ml}$ leupeptin, and $1 \mathrm{mM}$ PMSF) at $4^{\circ} \mathrm{C}$ for 2 hours with rotation. Immunoprecipitated proteins were separated by SDS-PAGE and then transferred to a nitrocellulose membrane. The membrane was blocked with TBS containing 5\% nonfat milk and $0.1 \%$ Tween 20 (TBST) at room temperature for 1 hour, followed by the incubation with primary antibody at $4^{\circ} \mathrm{C}$ overnight, and with the secondary antibody at room temperature for 1 hour. After washing with TBST, the membrane was developed using the enhanced chemiluminescent (ECL) detection system.

Immunohistochemistry. Immunohistochemistry was performed by using the peroxidase protocol. Briefly, tissue sections were deparaffinized in xylene, hydrated through descending ethanol, and endogenous peroxidase activity was eliminated by incubation in 3\% hydrogen peroxide in methanol for 5 minutes. After antigen retrieval in boiling sodium citrate buffer $(10 \mathrm{mM})$, the sections were incubated with primary antibodies overnight at $4^{\circ} \mathrm{C}$. The signal was developed using the Histostain-SP kit.

Quantification of colocalization. Pearson's coefficient, which is a standard statistical analysis to measure the strength of a linear relationship between 2 variables, was used to analyze the colocalization in duallabeled immunofluorescence images. We used ImageJ (NIH) to perform quantitative analysis of confocal images. Pearson's correlation coefficients were quantified by the 'Colocalization' tool in ImageJ. The values were set between 0 and 1 ( 1 = colocalization, $0=$ no colocalization).

Electron microscopy. After deep anesthesia, mice were perfused transcardially with $2 \%$ glutaraldehyde and 3\% paraformaldehyde in PBS. Hippocampal slices were postfixed in cold $1 \% \mathrm{OsO}_{4}$ for 1 hour. Samples were prepared and examined using standard procedures. Ultrathin sections $(90 \mathrm{~nm})$ were stained with uranyl acetate and lead acetate and viewed at $100 \mathrm{kV}$ in a JEOL 200CX electron microscope. Synapses were identified by the presence of synaptic vesicles and postsynaptic densities.

Stereotaxic injection of virus into the $S N$. Animals were anesthetized with isoflurane (Piramal Healthcare). Meloxicam (2 mg/kg) was injected subcutaneously for analgesics (Loxicom, Norbrook). Unilateral intracerebral injection of virus was performed stereotaxically at the following coordinates: anteroposterior (AP) $-3.1 \mathrm{~mm}$ and mediolateral (ML) $-1.2 \mathrm{~mm}$ relative to bregma, and dorsoventral (DV) $-4.3 \mathrm{~mm}$ from the dural surface. Viral suspension ( $2 \mu 1)$ was injected into each site using a 10- $\mu$ l Hamilton syringe with a fixed needle at a rate of $0.25 \mu 1 / \mathrm{min}$. The needle remained in place for 5 minutes after the viral suspension was completely injected at which point the needle was removed slowly (over 2 minutes). The mice were placed on a heating pad until they began to recover from the anesthesia. 
Electrophysiology. Acute hippocampal transversal slices were prepared from Tau P301S mice injected with lentivirus. Briefly, mice were anaesthetized with isoflurane, decapitated, and their brains dropped in ice-cold artificial cerebrospinal fluid (a-CSF) containing $124 \mathrm{mM} \mathrm{NaCl}, 3 \mathrm{mM} \mathrm{KCl}, 1.25 \mathrm{mM} \mathrm{NaH} \mathrm{PO}_{4}, 6.0 \mathrm{mM}$ $\mathrm{MgCl}_{2}, 26 \mathrm{mM} \mathrm{NaHCO}, 2.0 \mathrm{mM} \mathrm{CaCl}$, and $10 \mathrm{mM}$ glucose. Hippocampi were dissected and cut into $400-\mu \mathrm{m}$-thick transverse slices with a vibratome. The field excitatory postsynaptic potentials (fEPSPs) were recorded in the CA1 stratum radiatum by a glass microelectrode filled with a-CSF with resistance of 3 to 4 $\mathrm{M} \Omega$. fEPSPs were recorded under current-clamp mode. Data were filtered at $3 \mathrm{kHz}$ and digitized at sampling rates of $20 \mathrm{kHz}$ using Pulse software (HEKA Elektronik). fEPSP and the test pulse was applied at a rate of $0.033 \mathrm{~Hz}$. LTP of fEPSPs was induced by 3 -theta-burst stimulation (4 pulses at $100 \mathrm{~Hz}$, repeated 3 times with a 200-ms interval). The magnitudes of LTP are expressed as the mean percentage of baseline fEPSP initial slope.

Statistics. All data are expressed as mean \pm SEM from 3 or more independent experiments, and the level of significance between 2 groups was assessed with an unpaired 2-tailed $t$ test. For more than 2 groups, 1-way or 2-way ANOVA was applied. A value of $P<0.05$ was considered to be statistically significant.

Study approval. Animal care and handling was performed according to NIH animal care guidelines and Emory Medical School guidelines. The protocol was reviewed and approved by the Emory Institutional Animal Care and Use Committee.

\section{Author contributions}

KY conceived the project, designed the experiments, and wrote the manuscript. ZHW designed and performed most of the experiments. WW, SSK, and XL (Xia Liu) prepared primary neurons and assisted with animal experiments. IMS and FPM provided clones and packaged viral vectors. ZW and JP performed the mass spectrometry analysis. ZHW, SPY, FPM, XL (Xuebo Liu), and JZW assisted with data analysis and interpretation and critically read the manuscript.

\section{Acknowledgments}

This work was supported by NIH grants RF1 AG051538 (to K. Ye) and and R01 AG047928 (to J. Peng); and National Nature Science Foundation of China (NSFC) grant (no. 81528007) (to K. Ye and J.Z. Wang). We thank the Alzheimer's Disease Research Center at Emory University for human patients and healthy control samples. This study was supported in part by the Rodent Behavioral Core (RBC), which is subsidized by the Emory University School of Medicine and is one of the Emory Integrated Core Facilities.

Address correspondence to: Keqiang Ye, 141 Whitehead Building, Department of Pathology and Laboratory Medicine, Emory University School of Medicine, 615 Michael Street, Atlanta, Georgia 30322, USA. Phone: 404.712.2814; Email: kye@emory.edu. Or to: Jian-Zhi Wang, Department of Pathophysiology, Tongji Medical College, Huazhong University of Science and Technology, 13 Hangkong Road, 430030 Wuhan, China. Phone: 86.27.83693883; Email: wangjz@mails.tjmu.edu.cn.

1. Lin MT, Beal MF. Mitochondrial dysfunction and oxidative stress in neurodegenerative diseases. Nature. 2006;443(7113):787-795.

2. Zhang Z, et al. Delta-secretase cleaves amyloid precursor protein and regulates the pathogenesis in Alzheimer's disease. Nat Commun. 2015;6:8762.

3. Zhang Z, et al. Cleavage of tau by asparagine endopeptidase mediates the neurofibrillary pathology in Alzheimer's disease. Nat Med. 2014;20(11):1254-1262.

4. Zhang Z, et al. Asparagine endopeptidase cleaves $\alpha$-synuclein and mediates pathologic activities in Parkinson's disease. Nat Struct Mol Biol. 2017;24(8):632-642.

5. Zhang Z, et al. Inhibition of delta-secretase improves cognitive functions in mouse models of Alzheimer's disease. Nat Commun. 2017;8:14740

6. Herskowitz JH, et al. Asparaginyl endopeptidase cleaves TDP-43 in brain. Proteomics. 2012;12(15-16):2455-2463.

7. Liu Z, et al. Neuroprotective actions of PIKE-L by inhibition of SET proteolytic degradation by asparagine endopeptidase. $M o l$ Cell. 2008;29(6):665-678.

8. Basurto-Islas G, Grundke-Iqbal I, Tung YC, Liu F, Iqbal K. Activation of asparaginyl endopeptidase leads to Tau hyperphosphorylation in Alzheimer disease. J Biol Chem. 2013;288(24):17495-17507.

9. Li DN, Matthews SP, Antoniou AN, Mazzeo D, Watts C. Multistep autoactivation of asparaginyl endopeptidase in vitro and in vivo. J Biol Chem. 2003;278(40):38980-38990.

10. Maehr R, et al. Asparagine endopeptidase is not essential for class II MHC antigen presentation but is required for processing of cathepsin L in mice. J Immunol. 2005;174(11):7066-7074.

11. Shirahama-Noda K, et al. Biosynthetic processing of cathepsins and lysosomal degradation are abolished in asparaginyl endopeptidase-deficient mice. J Biol Chem. 2003;278(35):33194-33199. 
12. Chan CB, et al. Mice lacking asparaginyl endopeptidase develop disorders resembling hemophagocytic syndrome. Proc Natl Acad Sci USA. 2009;106(2):468-473.

13. Qi Q, Obianyo O, Du Y, Fu H, Li S, Ye K. Blockade of asparagine endopeptidase inhibits cancer metastasis. J Med Chem. 2017;60(17):7244-7255.

14. Wang ZH, et al. Delta-secretase phosphorylation by SRPK2 Enhances its enzymatic activity, provoking pathogenesis in Alzheimer's disease. Mol Cell. 2017;67(5):812-825.e5.

15. Balaratnasingam S, Janca A. Brain derived neurotrophic factor: a novel neurotrophin involved in psychiatric and neurological disorders. Pharmacol Ther. 2012;134(1):116-124.

16. Huang EJ, Reichardt LF. Neurotrophins: roles in neuronal development and function. Annu Rev Neurosci. 2001;24:677-736.

17. Bartoletti A, et al. Heterozygous knock-out mice for brain-derived neurotrophic factor show a pathway-specific impairment of long-term potentiation but normal critical period for monocular deprivation. J Neurosci. 2002;22(23):10072-10077.

18. Gorski JA, Zeiler SR, Tamowski S, Jones KR. Brain-derived neurotrophic factor is required for the maintenance of cortical dendrites. J Neurosci. 2003;23(17):6856-6865.

19. Heldt SA, Stanek L, Chhatwal JP, Ressler KJ. Hippocampus-specific deletion of BDNF in adult mice impairs spatial memory and extinction of aversive memories. Mol Psychiatry. 2007;12(7):656-670.

20. Zuccato C, Cattaneo E. Brain-derived neurotrophic factor in neurodegenerative diseases. Nat Rev Neurol. 2009;5(6):311-322.

21. Diniz BS, Teixeira AL. Brain-derived neurotrophic factor and Alzheimer's disease: physiopathology and beyond. Neuromolecular Med. 2011;13(4):217-222.

22. Murer MG, Yan Q, Raisman-Vozari R. Brain-derived neurotrophic factor in the control human brain, and in Alzheimer's disease and Parkinson's disease. Prog Neurobiol. 2001;63(1):71-124.

23. Peng S, et al. Decreased brain-derived neurotrophic factor depends on amyloid aggregation state in transgenic mouse models of Alzheimer's disease. J Neurosci. 2009;29(29):9321-9329.

24. Ventriglia M, et al. Serum brain-derived neurotrophic factor levels in different neurological diseases. Biomed Res Int. 2013;2013:901082.

25. Murer MG, et al. An immunohistochemical study of the distribution of brain-derived neurotrophic factor in the adult human brain, with particular reference to Alzheimer's disease. Neuroscience. 1999;88(4):1015-1032.

26. Ando S, et al. Animal model of dementia induced by entorhinal synaptic damage and partial restoration of cognitive deficits by BDNF and carnitine. J Neurosci Res. 2002;70(3):519-527.

27. Arancibia S, et al. Protective effect of BDNF against beta-amyloid induced neurotoxicity in vitro and in vivo in rats. Neurobiol Dis. 2008;31(3):316-326.

28. Nagahara AH, et al. Neuroprotective effects of brain-derived neurotrophic factor in rodent and primate models of Alzheimer's disease. Nat Med. 2009;15(3):331-337.

29. Kohno R, Sawada H, Kawamoto Y, Uemura K, Shibasaki H, Shimohama S. BDNF is induced by wild-type alpha-synuclein but not by the two mutants, A30P or A53T, in glioma cell line. Biochem Biophys Res Commun. 2004;318(1):113-118.

30. Klein RL, Lewis MH, Muzyczka N, Meyer EM. Prevention of 6-hydroxydopamine-induced rotational behavior by BDNF somatic gene transfer. Brain Res. 1999;847(2):314-320.

31. Levivier M, Przedborski S, Bencsics C, Kang UJ. Intrastriatal implantation of fibroblasts genetically engineered to produce brain-derived neurotrophic factor prevents degeneration of dopaminergic neurons in a rat model of Parkinson's disease. J Neurosci. 1995;15(12):7810-7820.

32. Yoshimoto Y, Lin Q, Collier TJ, Frim DM, Breakefield XO, Bohn MC. Astrocytes retrovirally transduced with BDNF elicit behavioral improvement in a rat model of Parkinson's disease. Brain Res. 1995;691(1-2):25-36

33. Frim DM, Uhler TA, Galpern WR, Beal MF, Breakefield XO, Isacson O. Implanted fibroblasts genetically engineered to produce brain-derived neurotrophic factor prevent 1-methyl-4-phenylpyridinium toxicity to dopaminergic neurons in the rat. Proc Natl Acad Sci USA. 1994;91(11):5104-5108

34. Brix K, McInnes J, Al-Hashimi A, Rehders M, Tamhane T, Haugen MH. Proteolysis mediated by cysteine cathepsins and legumain-recent advances and cell biological challenges. Protoplasma. 2015;252(3):755-774.

35. Lunde NN, et al. Glycosylation is important for legumain localization and processing to active forms but not for cystatin E/M inhibitory functions. Biochimie. 2017;139:27-37.

36. Manoury B, Hewitt EW, Morrice N, Dando PM, Barrett AJ, Watts C. An asparaginyl endopeptidase processes a microbial antigen for class II MHC presentation. Nature. 1998;396(6712):695-699.

37. Yoshiyama Y, et al. Synapse loss and microglial activation precede tangles in a P301S tauopathy mouse model. Neuron. 2007;53(3):337-351.

38. Janezic S, et al. Deficits in dopaminergic transmission precede neuron loss and dysfunction in a new Parkinson model. Proc Natl Acad Sci USA. 2013;110(42):E4016-E4025.

39. Ginsberg SD, et al. Microarray analysis of hippocampal CA1 neurons implicates early endosomal dysfunction during Alzheimer's disease progression. Biol Psychiatry. 2010;68(10):885-893.

40. Peng S, Wuu J, Mufson EJ, Fahnestock M. Precursor form of brain-derived neurotrophic factor and mature brain-derived neurotrophic factor are decreased in the pre-clinical stages of Alzheimer's disease. J Neurochem. 2005;93(6):1412-1421.

41. Connor B, Young D, Yan Q, Faull RL, Synek B, Dragunow M. Brain-derived neurotrophic factor is reduced in Alzheimer's disease. Brain Res Mol Brain Res. 1997;49(1-2):71-81.

42. Tapia-Arancibia L, Aliaga E, Silhol M, Arancibia S. New insights into brain BDNF function in normal aging and Alzheimer disease. Brain Res Rev. 2008;59(1):201-220.

43. Mogi M, et al. Brain-derived growth factor and nerve growth factor concentrations are decreased in the substantia nigra in Parkinson's disease. Neurosci Lett. 1999;270(1):45-48.

44. Parain K, et al. Reduced expression of brain-derived neurotrophic factor protein in Parkinson's disease substantia nigra. Neuroreport. 1999;10(3):557-561.

45. Akatsu H, et al. Variations in the BDNF gene in autopsy-confirmed Alzheimer's disease and dementia with Lewy bodies in Japan. Dement Geriatr Cogn Disord. 2006;22(3):216-222. 
46. Huang R, Huang J, Cathcart H, Smith S, Poduslo SE. Genetic variants in brain-derived neurotrophic factor associated with Alzheimer's disease. J Med Genet. 2007;44(2):e66

47. Olin D, MacMurray J, Comings DE. Risk of late-onset Alzheimer's disease associated with BDNF C270T polymorphism. Neurosci Lett. 2005;381(3):275-278.

48. Momose Y, et al. Association studies of multiple candidate genes for Parkinson's disease using single nucleotide polymorphisms. Ann Neurol. 2002;51(1):133-136.

49. Parsian A, Sinha R, Racette B, Zhao JH, Perlmutter JS. Association of a variation in the promoter region of the brain-derived neurotrophic factor gene with familial Parkinson's disease. Parkinsonism Relat Disord. 2004;10(4):213-219.

50. Galpern WR, Frim DM, Tatter SB, Altar CA, Beal MF, Isacson O. Cell-mediated delivery of brain-derived neurotrophic factor enhances dopamine levels in an $\mathrm{MPP}^{+}$rat model of substantia nigra degeneration. Cell Transplant. 1996;5(2):225-232

51. Castello NA, Green KN, LaFerla FM. Genetic knockdown of brain-derived neurotrophic factor in $3 \times$ Tg-AD mice does not alter A $\beta$ or tau pathology. PLoS One. 2012;7(8):e39566.

52. Devi L, Ohno M. TrkB reduction exacerbates Alzheimer's disease-like signaling aberrations and memory deficits without affecting $\beta$-amyloidosis in 5XFAD mice. Transl Psychiatry. 2015;5:e562.

53. Matrone C, Ciotti MT, Mercanti D, Marolda R, Calissano P. NGF and BDNF signaling control amyloidogenic route and Abeta production in hippocampal neurons. Proc Natl Acad Sci USA. 2008;105(35):13139-13144.

54. Rohe M, Synowitz M, Glass R, Paul SM, Nykjaer A, Willnow TE. Brain-derived neurotrophic factor reduces amyloidogenic processing through control of SORLA gene expression. J Neurosci. 2009;29(49):15472-15478.

55. Elliott E, Atlas R, Lange A, Ginzburg I. Brain-derived neurotrophic factor induces a rapid dephosphorylation of tau protein through a PI-3 Kinase signalling mechanism. Eur J Neurosci. 2005;22(5):1081-1089.

56. Husseman JW, Nochlin D, Vincent I. Mitotic activation: a convergent mechanism for a cohort of neurodegenerative diseases. Neurobiol Aging. 2000;21(6):815-828

57. Jang SW, et al. Interaction of Akt-phosphorylated SRPK2 with 14-3-3 mediates cell cycle and cell death in neurons. J Biol Chem. 2009;284(36):24512-24525.

58. Zhao L, et al. Structural analysis of asparaginyl endopeptidase reveals the activation mechanism and a reversible intermediate maturation stage. Cell Res. 2014;24(3):344-358.

59. Dall E, Brandstetter H. Mechanistic and structural studies on legumain explain its zymogenicity, distinct activation pathways, and regulation. Proc Natl Acad Sci USA. 2013;110(27):10940-10945.

60. Jadhav S, Zilka N, Novak M. Protein truncation as a common denominator of human neurodegenerative foldopathies. Mol Neurobiol. 2013;48(3):516-532.

61. Pagala VR, et al. Quantitative protein analysis by mass spectrometry. Methods Mol Biol. 2015;1278:281-305.

62. Wang X, Li Y, Wu Z, Wang H, Tan H, Peng J. JUMP: a tag-based database search tool for peptide identification with high sensitivity and accuracy. Mol Cell Proteomics. 2014;13(12):3663-3673. 